\title{
Real-Time Optimization of an Industrial Steam-Methane Reformer Under Distributed Sensing
}

\author{
Ankur Kumar ${ }^{\mathrm{a}}$, Michael Baldea ${ }^{\mathrm{a}, *}$, Thomas F. Edgar ${ }^{\mathrm{a}}$ \\ ${ }^{a}$ McKetta Department of Chemical Engineering, The University of Texas at Austin, Austin, TX 78712
}

\begin{abstract}
Industrial hydrogen production takes place in large-scale steam methane reformer (SMR) units, whose energy efficiency depends on the interior spatial temperature distribution. In this paper, a control-relevant empirical reduced-order SMR model is presented that predicts the furnace temperature distribution based on fuel input to a group of burners. The model is calibrated using distributed temperature measurements from an array of infrared cameras. The model is employed to optimize in real-time the temperature distribution and increase the energy efficiency in an industrial furnace. Experimental results confirm that the proposed framework has excellent performance.

Keywords: Reduced order modeling, Control of distributed parameter systems, Steam methane reformer, Smart Manufacturing
\end{abstract}

\section{Introduction}

Hydrogen $\left(\mathrm{H}_{2}\right)$ is a bulk chemical required for manufacturing many important products. For example, it is an essential component of the feedstock for ammonia, methanol, and other commodity chemicals. It is also consumed in large quantities in refineries for processing heavy or sour (high sulfur content) crude oil. Increased demands in these applications mean that demand for hydrogen will continue to rise. $\frac{1}{2}$ The dominant source of $\mathrm{H}_{2}$ for such industrial purposes is methane-based steam reforming. The reaction sequence includes endothermic reaction of natural gas (methane) and the

\footnotetext{
* Corresponding author

Email address: mbaldea@che.utexas. edu (Michael Baldea)
}

(C) 2016. This manuscript version is made available under the Elsevier user license http://www.elsevier.com/open-access/userlicense/1.0/ 
exothermic water-gas shift reaction (Eq. (1)):

$$
\begin{array}{rr}
\mathrm{CH}_{4}(g)+2 \mathrm{H}_{2} \mathrm{O}(g) \leftrightarrow 4 H_{2}(g)+\mathrm{CO}_{2}(g), & \triangle H_{298 K}=165 \mathrm{KJ} / \mathrm{mol} \\
\mathrm{CH}_{4}(g)+\mathrm{H}_{2} \mathrm{O}(g) \leftrightarrow 3 H_{2}(g)+C O(g), & \triangle H_{298 K}=206 \mathrm{KJ} / \mathrm{mol} \\
\mathrm{CO}(g)+\mathrm{H}_{2} \mathrm{O}(g) \leftrightarrow \mathrm{CO}_{2}(g)+H_{2}(g), & \triangle H_{298 K}=-41.2 \mathrm{KJ} / \mathrm{mol}
\end{array}
$$

These energy-intensive reactions take place in catalyst-filled tubes placed in a physically large-scale refractory-lined furnace called a steam methane reformer (SMR). Schematic 1 shows a typical hydrogen manufacturing plant, where synthesis gas product from the SMR passes through a shift reactor followed by product $\left(\mathrm{H}_{2}\right)$ separation via pressure swing adsorption (PSA). The SMR unit has two distinct regions (see Figure 2(a) . The process reactants flow inside the catalyst-packed reformer tubes while the exhaust gases, product of combustion of a mixture of natural gas feed and hydrogenrich PSA-bed recycle, flow outside the tubes. Energy released through the air-assisted combustion supports the endothermic reforming reactions.

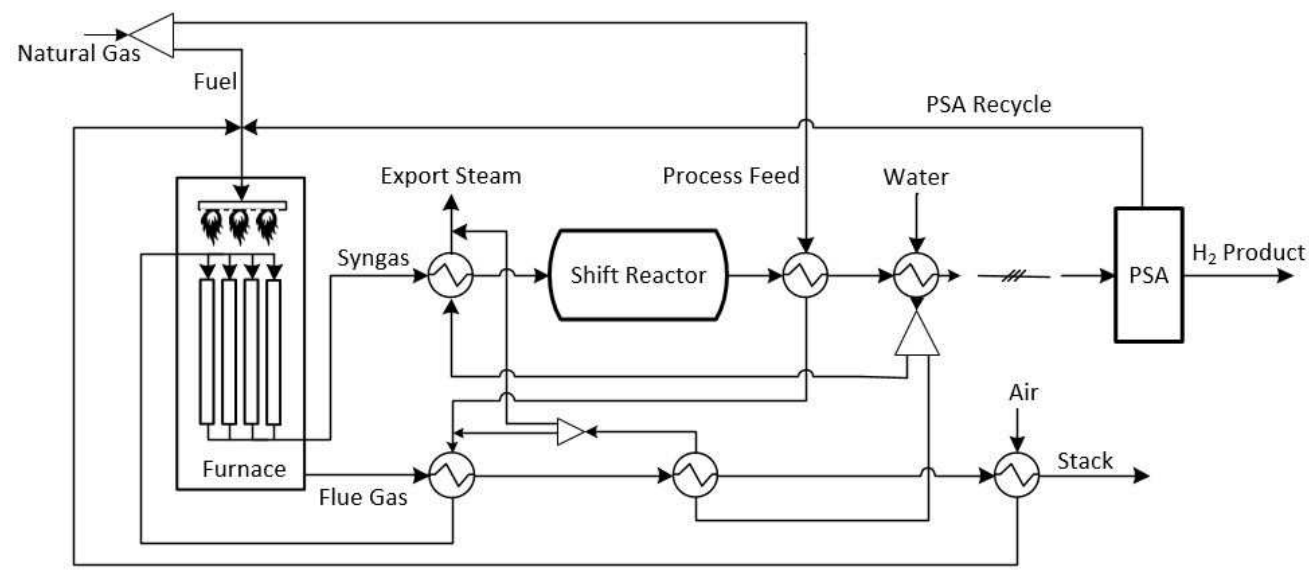

Figure 1: Schematic of a reforming-based hydrogen production plant (adapted from Esposito and Dadebo ${ }^{2}$ )

A typical modern hydrogen production plant of capacity $112,000 \mathrm{Nm}^{3} / h(\sim 100$ million standard cubic feet per day) of $\mathrm{H}_{2}$ consumes a substantial amount $\left(\sim 10^{5}\right.$ $G J)$ of natural gas per day. $\frac{3}{-}$ Consequently, the overall energy productivity (energy consumed per unit $\mathrm{H}_{2}$ produced) of the plant is strongly dependent on how efficiently the SMR is operated. In the spatially-distributed high temperature environment, the 
temperature of the exhaust gas is typically in the $\sim 1300 \mathrm{~K}$ range,, 4 of the furnace, one way to ensure maximum operation efficiency is to process the entire feedstock in the most similar way in order to make uniform products with minimum waste. $\frac{5}{}$ This translates to maintaining a uniform spatial tube temperature profile within the furnace. Note that, temperature variations along the length, from top to bottom, of a tube are inevitable. However, in an ideal scenario, the axial tube temperature profile for every tube is the same, i.e., at a given axial position (furnace height), tube wall temperatures for all the tubes would be identical. The temperature distribution depends on operating conditions such as the feed flow rate through the reformer tubes, ambient temperature, and fuel and air distribution among the burners, as well as on design of the burners and the fuel and air distribution header. In practice, as reported by Slavejkov et al., $\underline{\underline{6}}$ the discrepancy between the maximum and minimum tube-wall temperatures (TWTs) at a given axial position can be as high as $110 \mathrm{~K}$.

A practical approach for real-time control of the temperature distribution is through manipulation of fuel distribution among the burners. Figure 3 shows the fuel distribution system where the burners draw fuel from a fuel header. All the fuel headers supply fuel to burners in their respective rows and are joined to a main inlet fuel header at one end of the furnace. The fuel stream for each burner is typically provided with a manual valve and thus fuel from a burner can be diverted to the other burners by partially closing the valve. However, the TWTs and thus the optimal fuel distribution change due to disturbances that affect the furnace. Thus the optimal fuel distribution needs to be adjusted periodically since there is no unique optimal fuel distribution that works for all operating conditions. This in turn requires a sufficiently accurate furnace model that can predict in real-time the TWT distribution as a function of the fuel distribution or any other manipulated variable that indirectly influences the fuel distribution. Most of the first-principles-based furnace models ${ }^{1,4,7-11}$ available in the literature do not resolve the complete TWT distribution. Either all the tubes are lumped together to give an average tube temperature, or only the tubes in different radiative environments are modeled separately 11 . Consequently, Dunn et al. ${ }^{11}$ carried out partial furnace optimization by adjusting fuel and reactant gas distribution. Apart from tube lumping, several other approximations are made, such as ignoring the impact of flue gas flow 


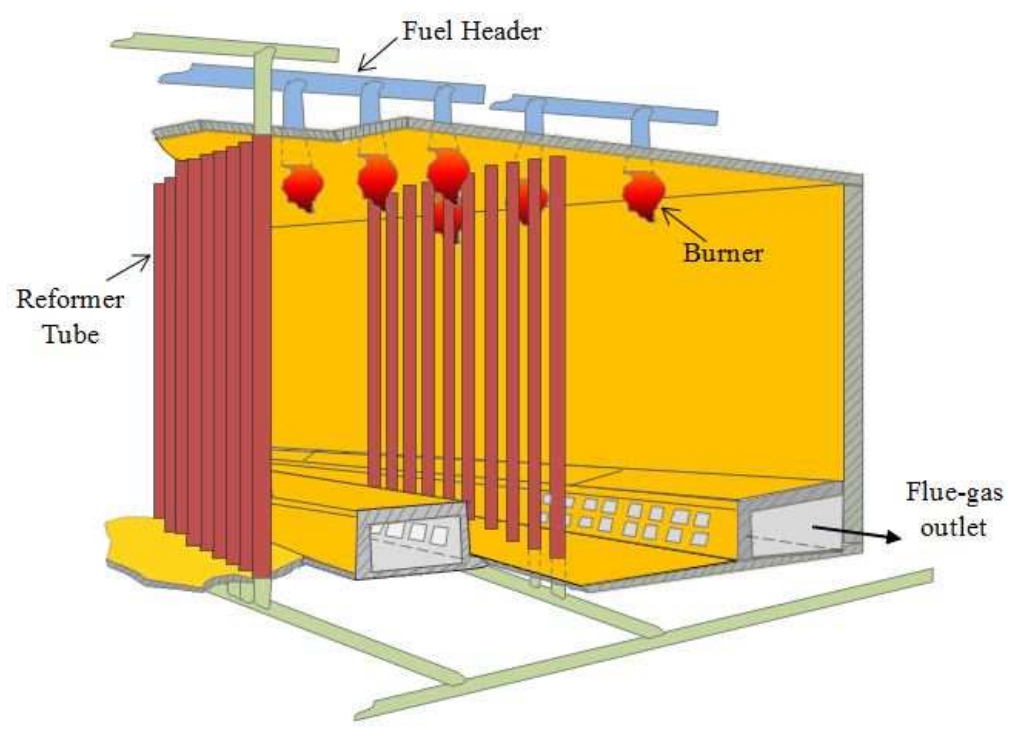

(a)

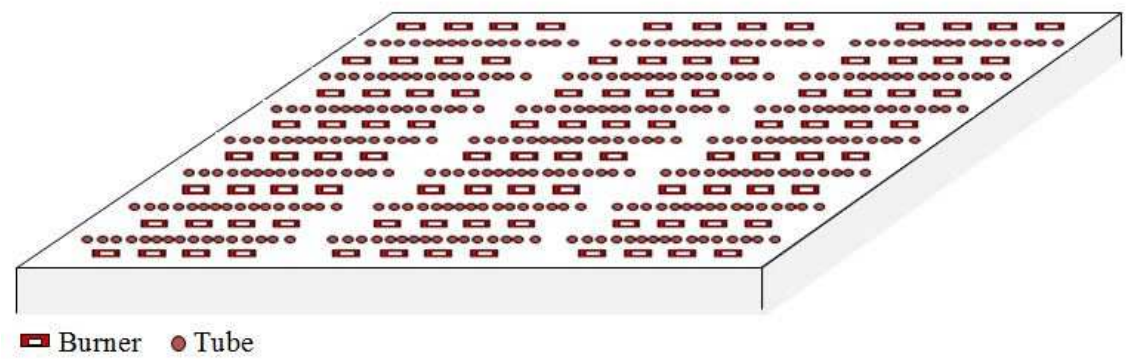

(b)

Figure 2: Schematic of (a) a typical top-fired SMR and (b) top-view showing the arrangements of burners and tubes in the test-bed SMR with 8 and 7 rows of burners and tubes, respectively (adapted from Latham et al. $\stackrel{7}{=})$

patterns, which can lead to inaccurate TWT predictions $\frac{12,13}{2}$. Computational fluid dynamics (CFD) modeling provides an alternative for rigorous analysis of phenomena occurring within an SMR. Using CFD to resolve the realistic flow field in a coupled

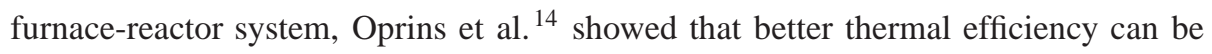
obtained by non-uniform fuel gas distribution. Zheng et al. $\stackrel{15}{\frac{15}{2}}$ showed that the tube temperature distribution is adversely affected by maldistribution of flue gas, leading to localized hot regions. However, these CFD models are computationally intensive and 


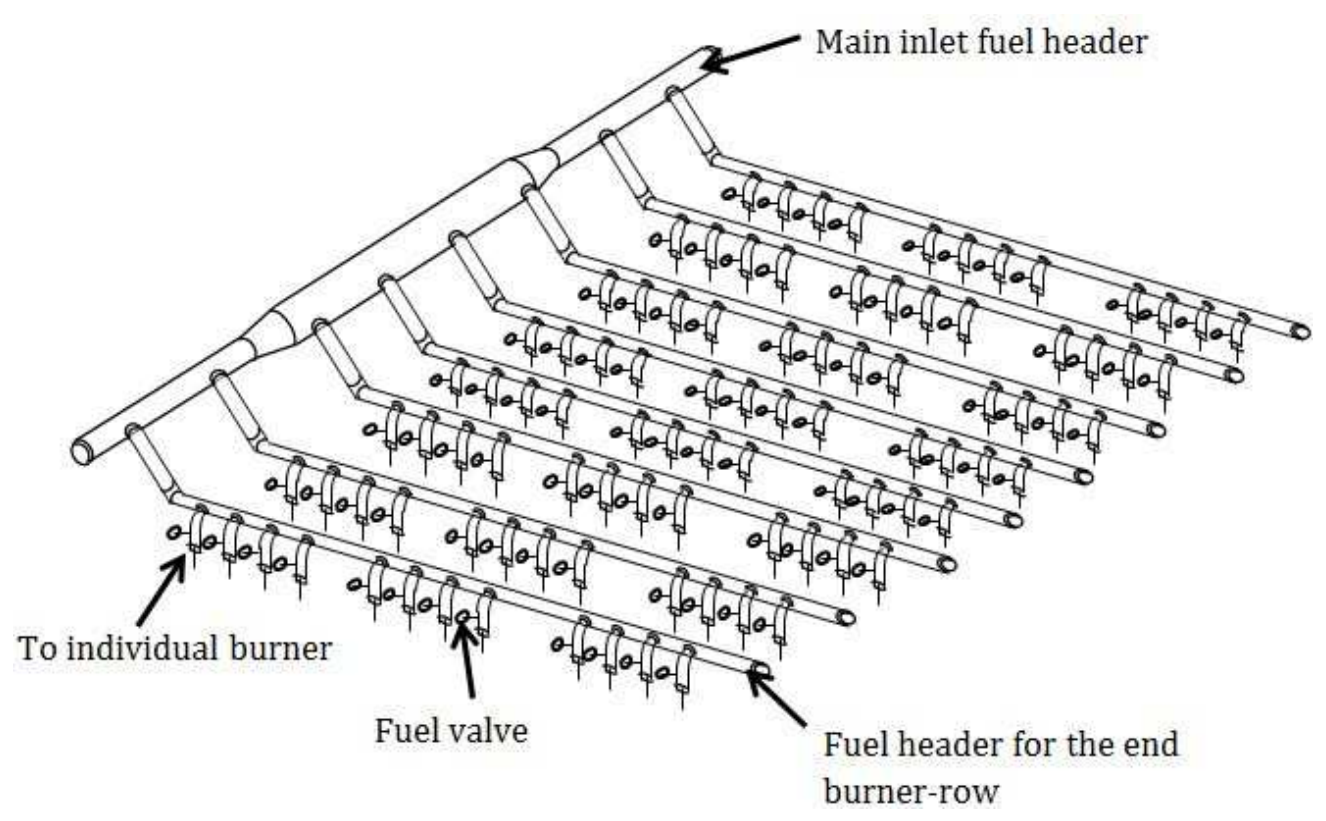

Figure 3: Schematic of the fuel header arrangement supplying fuel to individual burners

thus are unsuitable for real-time furnace optimization. Blevins and Nixon $\stackrel{16}{\underline{16}}$ describe a model-predictive control (MPC) implementation for control of reformer temperature. Here, the fuel flow rate through each row of burners is adjusted to control the lumped outlet temperatures of multiple tube bundles. The temperature distribution within the furnace itself is not modeled or controlled. Slavejkov et al. $\underline{\underline{6}}$ attempted heuristics-based manual fuel flow adjustments to decrease the tube-to-tube temperature variability. The feasibility of using a data-driven empirical model to achieve uniform furnace temperature profile was demonstrated by Kumar et. al.. 17 A proper orthogonal decompositionbased reduced-order linear model was used to modulate the fuel distribution among the burners in a representative smaller-scale SMR model. It was argued that a reducedorder empirical model with much lower computational requirements, when trained with sufficiently rich data, can be a viable substitute to the detailed modeling of the complex thermal and flow interactions in the furnace using, e.g., CFD. In this paper, the data-driven modeling approach is extended to the full-scale SMR furnace. Furnace specific model characteristics have been incorporated to overcome the shortcomings in 
previously attempted approaches. $\frac{6,16,18}{}$ While it is common in industry to use process data to build empirical models based on methodologies such as ordinary least squares (OLS) regression or partial least squares (PLS) regression ${ }^{19}$, several limitations are encountered when these models are employed for approximating the relationship between the temperature changes and the fuel-stream valve manipulations in the furnace model. First, OLS / PLS models are linear and may not capture nonlinearities well and it is not clear how to appropriately customize these models to incorporate, if desired, the nonlinear interactions. Further limitations, such as not explicitly considering crucial aspects like fuel redistribution, are discussed in detail in the following sections. Moreover, due to fuel redistribution, the fuel-stream valve manipulations have both long-range effects (temperature changes observed far away from the manipulated burner) and short-range effects (temperature changes observed near the manipulated burner) on the tube temperature distribution. It is crucial to appropriately distinguish between these effects, particularly in the presence of noise in the temperature measurements, which is obscured in a OLS / PLS model. The Egg-Crate SMR (EC-SMR) model, presented in the current work, incorporates explicitly these crucial aspects and allows potential customization of model structure to enable a better fit with real-plant observations. The parameters of the EC-SMR model are estimated using furnace-wide temperature measurements. The temperatures are measured experimentally on an industrial furnace using online infrared pyrometry, a relatively new advance for SMRs.

The work presented in this paper is part of a Smart Manufacturing program 20 wherein industrial manufacturing solutions are provided and deployed on a cloudbased infrastructure. The program aims to facilitate greater adoption of advanced sensor-based data analytics and modeling in manufacturing plants, similar to the infrared imaging-assisted furnace optimization solution developed in this work. Several researchers ${ }^{21,22}$ have remarked on the need for greater embracement of cyberinfrastructure and data-driven knowledge-centric chemical plants towards realization of the 'Smart Plant' paradigm. The reader is referred to Edgar and Davis 23 and Davis et. $\mathrm{al}^{20}$ for a comprehensive description of the Smart Manufacturing framework.

This paper is structured as follows. In the next section the TWT distribution and its variations upon fuel-stream valve manipulation are discussed. The temperature uni- 
formization concept is briefly described. Then, the need for the EC-SMR model is motivated and its mathematical formulation is provided. This is followed by comparison of the EC-SMR model and PLS model predictions against the actual plant observations. Finally, the EC-SMR model is employed in mixed-integer optimization calculations aimed at achieving a uniform furnace temperature distribution in real-time.

\section{SMR Furnace: Temperature Distribution and Furnace Balancing}

Figure 2 shows the arrangement of tubes and burners in a typical top-fired furnace. The test-bed SMR considered in this study has lateral dimensions of $16 \mathrm{~m} \times 16 \mathrm{~m}$, with 336 tubes in seven rows of 48 tubes each, and 96 burners in eight rows of 12 burners each. Figure 4 shows the distribution of the tube-wall temperatures at a predetermined axial location, with all fuel-stream valves completely open. The reader is referred to Kumar et. al ${ }^{17}$ for a description of the measurement system, as well as for the rationale behind using the temperatures only at a predetermined axial elevation. The spatial temperature variability is evident in Figure 4 The minimization of this variability, termed 'furnace balancing', requires a judicious implementation of fuel-redistribution, i.e., the fuel-stream valves in the hotter regions of the furnace can be closed partially to divert the fuel to the other burners. It should be noted that in practice there is no measurement of each burner's fuel flow rate. Any fuel redistribution has to be realized through appropriate fuel-stream valve adjustments. The increments in temperatures upon closing any valve, as shown in Figure 11(b) in Appendix A, results from fuel diversion. It is thus important to appropriately account for the fuel redistribution while quantifying the impact of fuel-stream valve manipulation. This quantification is discussed in the following sections.

The temperature at which the furnace is operated, and the resulting TWT distribution, strongly influences the thermal efficiency of the SMR. Equation (2) shows the accepted way of quantifying the thermal efficiency $(\eta)$ of an SMR, $\underline{3}$

$$
\eta=\frac{E_{H_{2}}}{E_{N G}-Q_{\text {steam }}}
$$

where $E_{H_{2}}$ and $E_{N G}$ are the energy contents (usually the lower heating values) of the $\mathrm{H}_{2}$ product and the total natural gas fed into the system, respectively, and $Q_{\text {steam }}$ 


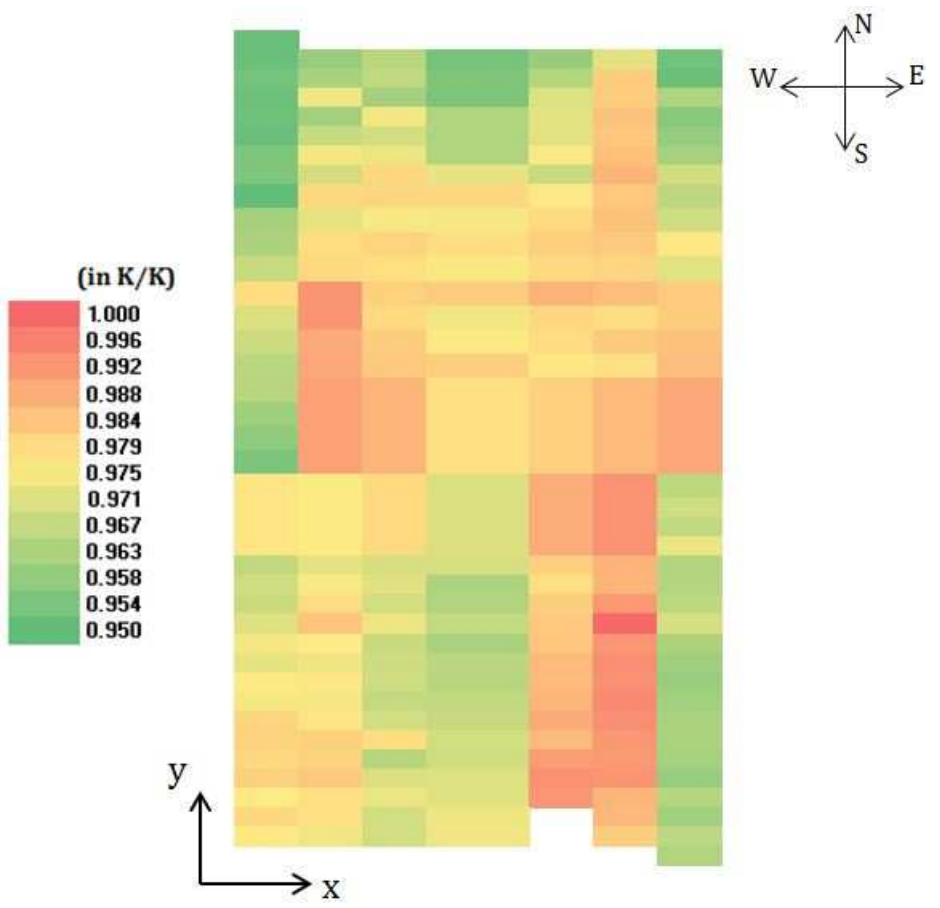

(a)

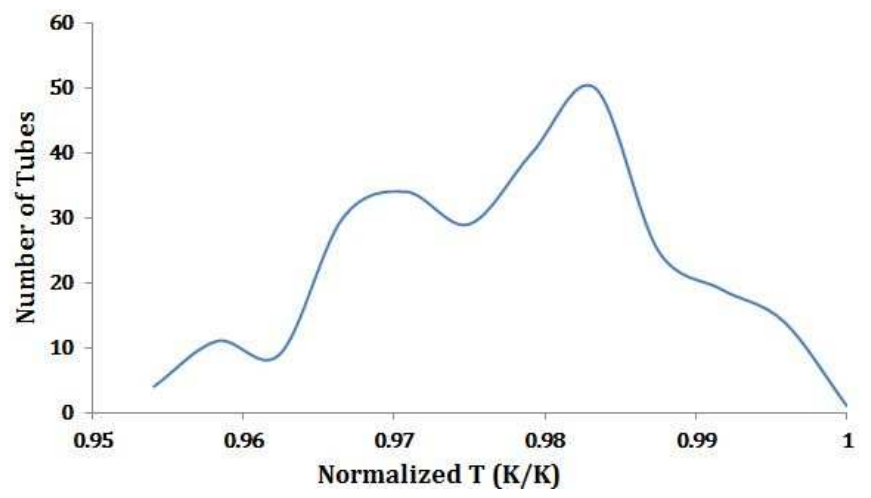

(b)

Figure 4: (a) Tube-wall temperatures at a fixed axial position obtained via IR imaging of a furnace under normal operation. Each colored unit corresponds to a tube. The shown reference coordinate system is explained in Section 3.2 Note that the reported temperatures have been normalized using the measured maximum TWT. (b) Histogram rendition of the distribution 
is the thermal value of the export-steam produced. The reforming reactions, being endothermic, attain higher methane conversion and $\mathrm{H}_{2}$ production at higher temperature. When the greater production and enthalpy of export steam, due to increased flue gas temperature (see Figure 1), are accounted for along with higher $\mathrm{H}_{2}$ yield, a lower overall energy consumption per unit $\mathrm{H}_{2}$ produced (or greater $\eta$ ) results. However, the furnace temperature is limited by the maximum operating temperature ( $T_{\max }$ in Figure 5) that the tubes are designed for. Exceeding the maximum temperature can potentially lead to tube ruptures because reformer tube life decreases sharply when operated above the design temperature. $\stackrel{7}{-}$ Any such failures entail significant economic costs due to replacement of damaged tubes, plant shut-downs and consequently production losses. However, furnace balancing or the reduction in the TWT nonuniformity allows for increasing the average furnace temperature through additional total fuel input, without violating the maximum temperature constraint (see Figure 5). Note that at a higher furnace temperature there is an alternate scenario (with greater $\eta$ ) where the $\mathrm{H}_{2}$ production is kept constant by reducing the total natural gas feed into the reformer tubes and leveraging the higher methane conversion. As pointed out by Latham et al. $\stackrel{7}{ }$, savings of $\$ 600,000 /$ year can be realized from a $1 \%$ reduction in energy costs for a 112,000 $N m^{3} / h \mathrm{H}_{2}$ plant.

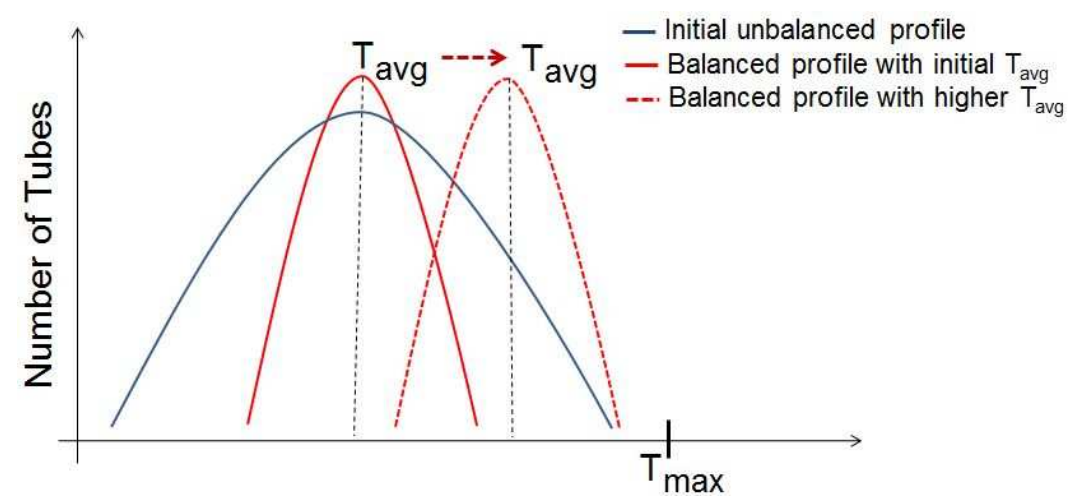

Figure 5: Furnace balancing (reduction in tube-wall temperature non-uniformity) followed by increase in average tube-wall temperature 


\section{Data-Driven Egg-Crate SMR Model}

\subsection{Motivation}

The furnace temperature distribution does not change significantly over a period of a few hours in absence of any major change in the operating conditions such as the plant production rate. Correspondingly, the valve adjustments for furnace balancing do not need to be made frequently. Hence, a steady-state relationship between the fuel-stream valves openings and the TWT distribution is of interest, and thus the dynamic effects are neglected here. To estimate such a relationship several experiments were performed on the commercial test-bed SMR. Each of these experiments involved partially closing some combinations of fuel-stream valves and then measuring the steady-state deviations in the tube-wall temperatures at a predetermined axial elevation, using the distributed temperature measurements continuously provided by the IR-cameras. Let $T_{i}, i \in\{1,2, \ldots, N\}$, represent the temperature of the $i^{t h}$ tube and $p_{j}, j \in\{1,2, \ldots, M\}$, represent the status of the $j^{t h}$ valve in terms of degrees open. $N$ is the number of tubes that are located in the field of view of the cameras and $M$ is the number of valves. A functional relationship of the following form is sought,

$$
\Delta \mathbf{T}=\mathbf{T}-\mathbf{T}_{0}=f(\Delta \mathbf{p})
$$

where $\mathbf{T}=\left[T_{1}, \ldots, T_{N}\right]^{T}$ is an $N$-dimensional vector representing the TWTs for the tubes, $\mathbf{T}_{0}$ is the initial TWT distribution, and $\Delta \mathbf{p}=\left[\Delta p_{1}, \ldots, \Delta p_{M}\right]^{T}$ is an $M$ dimensional vector representing the changes in the positions of the valves. It is assumed, without loss of generality, that all the valves are initially completely open. Ordinary least squares regression (OLSR) or partial least squares regression (PLSR) can be used to obtain a linear gain-matrix formulation,

$$
\Delta \mathrm{T}=\mathrm{G} \Delta \mathrm{p}
$$

where $\mathbf{G} \in \mathbb{R}^{N \times M}$. Each of its element $g_{i j}$ approximates the impact of closing or opening the $j^{\text {th }}$ valve on the $i^{\text {th }}$ tube temperature. While OLSR estimates the parameters, $g_{i j}$, directly by minimizing the difference between the experimental data and the linear model predictions (Eq. (4)), methods like PLSR make use of latent variables $\frac{19}{}$ for parameter estimation. 
There are some practical aspects that need to be considered during the selection and implementation of any particular modeling methodology. The laborious nature of data collection, apart from the demanding requirement of keeping the plant at or near a steady state, makes it difficult to conduct a large number of experiments. Thus, the valves were manipulated in groups of four (see Figure 11(a) in Appendix A providing a lower dimensional input-space for design of experiments 24 . Also the temperature measurements are corrupted, unavoidably, due to several disturbances such as fluctuations in fuel throughput or IR camera movements (see Figure 11(b) in Appendix A. The model should inherently be robust to such corrupted data. For example, in Figure 11(b) it is highly unlikely that the negative changes in the tube wall temperatures observed far away from the manipulated burners are true values. Tubes far away from the burners whose valves have been closed partially are expected to show only non-negative temperature changes. The model formulation should possess such characteristics. While the PLS models are known to be robust to noisy data, due to the limited number of experiments PLSR-based estimates remained sensitive to noise, as will be shown in Section 4 . Although the burners were experimentally manipulated in groups of four, the impact of individual valve adjustments is desired as well. The adjustments of valve-groups can provide a coarse furnace balancing, but individual valve changes are needed for finer balancing. Moreover, even though measurements are not available for the tubes that do not fall in the field of view of the cameras, it is desirable that the model be able to estimate the temperature changes for these tubes. The OLS / PLS model falls short vis-à-vis these requirements. Another crucial shortcoming is that Eq. (4) gives no information about what assumptions were made regarding fuel redistribution. Additionally, it might be necessary to explicitly model some important process features such as interaction effects of multiple valve adjustments on individual burner fuel-throughputs, interaction effects of multiple burner fuel-throughput changes on tube temperatures, relationship between valve adjustments and fuel-throughput changes. Explicit modeling of such features requires incorporating selective nonlinearities in the model structure.

The empirical furnace model, termed the egg-crate SMR (EC-SMR) model in this work, takes into account the above modeling requirements. The TWT deviations upon 
partially closing any $j^{\text {th }}$ valve are a combined effect of $j^{\text {th }}$ burner fuel-throughput reduction and fuel-throughput increments (the total fuel input to the furnace does not change) for all the other burners due to fuel redistribution. Figure 6(a) shows the combined effect as perturbations in the spatial temperature field (the observed TWT changes can be interpreted as discrete sampling of this infinite-dimensional field perturbation at spatial points where the tubes are located). Figure 6(b) shows the direct perturbations due to the $j^{\text {th }}$ burner fuel-throughput reduction and one of the multiple (95 to be precise) induced effects due to fuel-throughput increments. These effects can be combined linearly or nonlinearly to approximate the combined effect. The next sub-section represents this idea mathematically.

\subsection{Mathematical Formulation of EC-SMR Model}

Let $\tau_{i}, b_{j}$, and $v_{j}$ refer to the $i^{\text {th }}$ tube, the $j^{\text {th }}$ burner and the $j^{\text {th }}$ valve, respectively. The perturbations induced in the temperature field upon closing valve $v_{j}$ by 1 degree, represented as $\Delta p_{j}=1$, are first defined. Let $\overline{\Delta f}_{j}$ be the corresponding change in the fuel-throughput of the $j^{\text {th }}$ burner. The corresponding direct effect (see Figure 6(b) on tube $\tau_{i}$ is approximated using an exponentially decaying function as follows,

$$
\left.\Delta T_{i, j}\right|_{\Delta p_{j}=1}= \begin{cases}\overline{\Delta f}_{j} \times \beta_{j} e^{\left(-\frac{x_{i j}^{2} k_{x j}}{\sigma_{x j}^{2}}-\frac{y_{i j}^{2} k_{y j}}{\sigma_{y j}^{2}}\right)} & \text { if } x_{i j}>0, y_{i j}>0 \\ \overline{\Delta f}_{j} \times \beta_{j} e^{\left(-\frac{x_{i j}^{2}}{\sigma_{x j}^{2}}-\frac{y_{i j}^{2} k_{y j}}{\sigma_{y j}^{2}}\right)} & \text { if } x_{i j}<0, y_{i j}>0 \\ \overline{\Delta f}_{j} \times \beta_{j} e^{\left(-\frac{x_{i j}^{2} k_{x j}}{\sigma_{x j}^{2}}-\frac{y_{i j}^{2}}{\sigma_{y j}^{2}}\right)} & \text { if } x_{i j}>0, y_{i j}<0 \\ \overline{\Delta f}_{j} \times \beta_{j} e^{\left(-\frac{x_{i j}^{2}}{\sigma_{x j}^{2}}-\frac{y_{i j}^{2}}{\sigma_{y j}^{2}}\right)} & \text { if } x_{i j}<0, y_{i j}<0\end{cases}
$$

where $x_{i j}$ and $y_{i j}$ are the distance, between $\tau_{i}$ and $b_{j}$ along the $\mathrm{x}$-axis and the $\mathrm{y}$-axis, respectively. It should be noted that since temperatures only at a particular axial elevation are modeled, it is appropriate to use only two dimensions. Using the exponentially decaying local effects avoids over-fitting the long-range interactions during parameter estimation as will be shown later. The postulated expression in Eq. (5) is motivated by the experimental observations that the temperature changes are asymmetric, i.e., the 


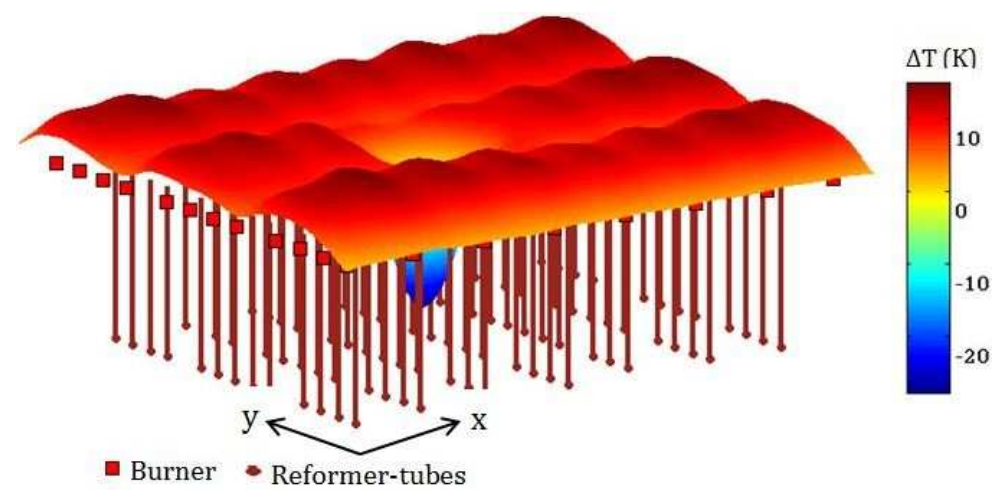

(a)
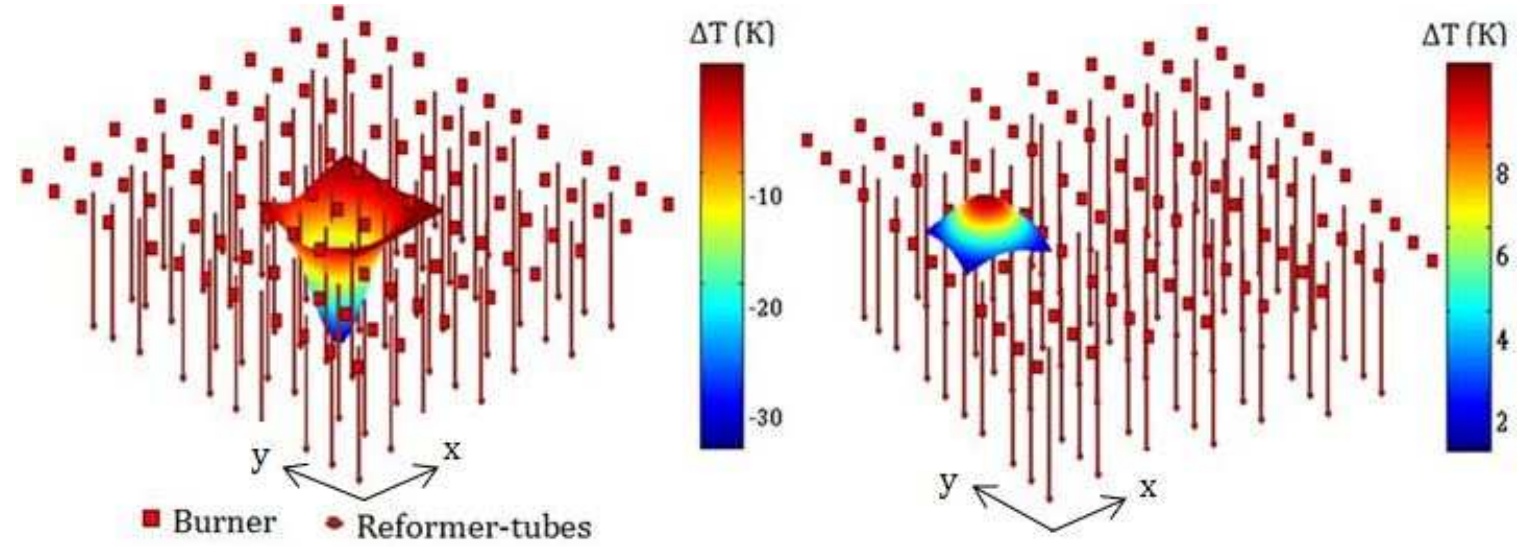

(b)

Figure 6: (a) Schematic of overall effect on tube-wall temperatures upon partially closing a valve. The values are exaggerated for illustration. A reduced number of tubes has been shown for clarity. The perturbations in the temperature field can be decomposed, as shown in Subfigure (b) into direct-effects (left) and multiple induced-effects (right)

temperature changes for the tubes along the tube rows on either side of the burner $b_{j}$, and at equal distances from the burner, were not necessarily equal (see Figure 11(b)). Since it was observed experimentally that valve-manipulations had varying effects depending on where the burner was located, every burner has been given six characteristic parameters, viz, $\overline{\Delta f}, \beta, \sigma_{x}, \sigma_{y}, k_{x}$, and $k_{y}$. $\beta$ denotes the maximum deviation produced by a burner in the temperature field per unit decrease in its fuel throughput. $\sigma_{x}$ and $\sigma_{y}$ are spread coefficients, which determine how far the direct effects are felt along the 
$\mathrm{x}$-axis and the $\mathrm{y}$-axis, respectively, and $k_{x}$ and $k_{y}$ are asymmetry coefficients, which allow the temperature changes to be asymmetric. One of the four cases shown in Eq. (5) is invoked depending upon whether the tube is located to the east or west and north or south of the burner (see Figure A.11). For convenience, the exponential term, called 'kernel', is represented as $\Phi_{i j}$, which is unique for a given pair of $\tau_{i}$ and $b_{j}$. Equation (5) can now compactly be written as,

$$
\left.\Delta T_{i, j}\right|_{\Delta p_{j}=1}=\overline{\Delta f}_{j} \times \beta_{j} \times \Phi_{i j}
$$

To define one of the multiple induced effects, the induced change in fuel throughput of burner $b_{k}\left(b_{k} \neq b_{j}\right)$, denoted as $\left.\Delta f_{k}\right|_{\Delta p_{j}=1}$, needs to be specified. The fuelredistribution model is incorporated here. Let parameter $\gamma_{j k}:=\left.\Delta f_{k}\right|_{\Delta p_{j}=1} / \overline{\Delta f}_{j}$, i.e., $\gamma_{j k}$ specifies the fraction of the fuel displaced from the burner $b_{j}$ that is redistributed to burner $b_{k}$. The induced effect on tube $\tau_{i}$ can now be defined as follows,

$$
\begin{aligned}
\left.\Delta T_{i, k}\right|_{\Delta p_{j}=1} & =\left.\Delta f_{k}\right|_{\Delta p_{j}=1} \times \beta_{k} \times \Phi_{i k} \quad \forall b_{k} \neq b_{j} \\
& =\gamma_{j k} \times \overline{\Delta f}_{j} \times \beta_{k} \times \Phi_{i k}
\end{aligned}
$$

Since the total fuel flow rate to the furnace does not change, $\gamma_{j k}$ are constrained to obey $\sum_{k=1, k \neq j}^{M} \gamma_{j k}=-1 \forall b_{j}$. The fuel redistribution parameters $\gamma_{j k}$ are assumed to be specified by the user. In this work, it is assumed that $\gamma_{j k}=\frac{-1}{M-1}$, i.e., the displaced fuel is uniformly redistributed to all the burners. Other schemes can also be easily implemented such as $\gamma_{j k}$ being inversely proportional to the distance between $b_{j}$ and $b_{k}$, i.e., the farther burner $b_{k}$ is from $b_{j}$, less fuel will be received. Note that the parameters $\gamma_{j k}$ could also be identified from a CFD simulation of only the fuel distribution header (as shown in Figure 3) without modeling the whole furnace. The direct effects (Eq. (6) and the induced effects (Eq. (7) can now be combined to give the overall impact. For this the following approximation is made,

$$
\left.\left.\Delta T_{i}\right|_{\Delta \mathbf{f}} \approx \sum_{k=1}^{M} \Delta T_{i}\right|_{\Delta f_{k}}
$$

where $\Delta \mathbf{f}=\left[\Delta f_{1}, \Delta f_{2}, \ldots, \Delta f_{M}\right]^{T}$ is an $M$-dimensional vector denoting the changes in fuel throughputs for the burners. Thus the combined effect is approximated as a 
linear combination of the direct and induced effects:

$$
\left.\Delta T_{i}\right|_{\Delta p_{j}=1}=\overline{\Delta f}_{j} \beta_{j} \Phi_{i j}+\sum_{k=1, k \neq j}^{M}-\frac{\overline{\Delta f}_{j}}{M-1} \beta_{k} \Phi_{i k}
$$

To compute the combined effect for a non-unit valve adjustment, i.e., $\Delta p_{j}>1$, a linear relationship between $\Delta p_{j}$ and $\Delta f_{j}$ is assumed. This can formally be postulated as,

$$
\left.\Delta f_{j}\right|_{\Delta p_{j}}=\Delta p_{j} \times \overline{\Delta f}_{j}
$$

Although a valve-opening vs fuel throughput curve generally has nonlinear characteristics, it can be reasonably approximated to be linear if $\Delta p$ is not very large. Note that $\Delta p_{j}=90$ degrees means that the $j^{\text {th }}$ burner is completely closed. Nonetheless, any suitable nonlinear behavior (for example, $\left.\Delta f_{j}\right|_{\Delta p_{j}}=\overline{\Delta f}_{j} \Delta p_{j}^{2}$ ) can be incorporated here, if desired, for specific cases.

Note that in Eq. (5) a linear relationship between the magnitude of a fuel-throughput change for any burner and the magnitude of temperature field perturbations was assumed. It is formally stated as,

$$
\left.\Delta T_{i}\right|_{\Delta f_{j}}=\Delta f_{j} \times \beta_{j} \times \Phi_{i j}
$$

While Eq. (8) ignores nonlinear cross-interactions, Eq. (11) imposes self-linearity for each individual burner. Note that, for any alternative functional form, Eqs. (6) and (7) will need to be modified accordingly. Combining Eqs. (9) and (10) gives the impact on tube $\tau_{i}$ for any $\Delta p_{j}$ change in valve position of burner $b_{j}$,

$$
\left.\Delta T_{i}\right|_{\Delta p_{j}}=\Delta p_{j} \times\left(\overline{\Delta f}_{j} \beta_{j} \Phi_{i j}+\sum_{k=1, k \neq j}^{M}-\frac{\overline{\Delta f}}{M-1} \beta_{k} \Phi_{i k}\right)
$$

Eq. (12) helps to understand the impact of simplifications such as imposing a common $\overline{\Delta f}_{j} \forall b_{j}$. For the common $\overline{\Delta f}_{j}$, consider two different experiments where burners $b_{j}$ and $b_{l}$ are closed partially, respectively. For a tube $\tau_{i},\left.\Delta T_{i}\right|_{\Delta p_{j}=1}=\left(\overline{\Delta f}_{j} \beta_{j} \Phi_{i j}+\right.$ $\left.\sum_{k=1, k \neq j}^{M}-\frac{\overline{\Delta f}_{j}}{M-1} \beta_{k} \Phi_{i k}\right)$ for the first experiment and similarly, for the second experiment $\left.\Delta T_{i}\right|_{\Delta p_{l}=1}=\left(\overline{\Delta f}_{l} \beta_{l} \Phi_{i l}+\sum_{k=1, k \neq l}^{M}-\frac{\overline{\Delta f_{l}}}{M-1} \beta_{k} \Phi_{i k}\right)$. Now, if the tube $\tau_{i}$ is far away from 
both burners $b_{j}$ and $b_{l}$, i.e., $\Phi_{i j} \sim 0$ and $\Phi_{i l} \sim 0$, then $\left.\Delta T_{i}\right|_{\Delta p_{j}=1}$ and $\left.\Delta T_{i}\right|_{\Delta p_{l}=1}$ become independent of the burner that has been closed partially and thus should be equal. However, such a prediction was not supported by the observations for the test-bed in this study.

Modeling the impact of manipulating any arbitrary combination of valves requires an understanding of how the fuel-throughputs change under such manipulations. Assuming $\left.\left.\Delta f_{j}\right|_{\Delta \mathbf{p}} \approx \sum_{k=1}^{M} \Delta f_{j}\right|_{\Delta p_{k}}$ and ignoring the cross-interactions among valve manipulations, the following can be written for general valve adjustments.

$$
\begin{aligned}
\left.\Delta T_{i}\right|_{\Delta \mathbf{p}} & =\sum_{k=1}^{M} \beta_{k} \Delta f_{k} \Phi_{i k} \quad \forall i \in\{1,2, \ldots, N\} \\
& =\sum_{k=1}^{M} \beta_{k}\left(\left.\sum_{j=1}^{M} \Delta f_{k}\right|_{\Delta p_{j}}\right) \Phi_{i k} \\
& =\left.\sum_{k=1}^{M} \sum_{j=1}^{M} \beta_{k} \Delta f_{k}\right|_{\Delta p_{j}} \Phi_{i k} \\
& =\left.\sum_{j=1}^{M} \sum_{k=1}^{M} \beta_{k} \Delta f_{k}\right|_{\Delta p_{j}} \Phi_{i k} \\
& =\left.\sum_{j=1}^{M} \Delta T_{i}\right|_{\Delta p_{j}} \\
\left.\Delta T_{i}\right|_{\Delta \mathbf{p}} & =\sum_{j=1}^{M} \Delta p_{j} \times\left(\overline{\Delta f}_{j} \beta_{j} \Phi_{i j}+\sum_{k=1, k \neq j}^{M}-\frac{\overline{\Delta f}_{j}}{M-1} \beta_{k} \Phi_{i k}\right) \quad \forall i \in\{1,2, \ldots, N\}
\end{aligned}
$$

The above expression describes the perturbations in the temperature field using the characteristic parameters assigned to each burner. It should be noted that Eq. (13) can be used to determine the temperature changes for the tubes that are not in the field of view of the cameras by using appropriate kernel $(\Phi)$ values.

Remark: The stated assumptions clearly show the avenues for model customization through incorporation of nonlinear behavior within the EC-SMR model formulation. A detailed CFD model, if available, of the SMR can be used to infer the appropriate nonlinear pattern and impose that on the EC-SMR model. For example, a different form for the kernel, $\Phi$, that determines the spatial shape of the produced temperature 
deviations, can be used if the alternative form matches the observed data better.

\subsection{Parameter Estimation via Nonlinear Optimization}

The EC-SMR model parameters are estimated by minimizing the differences between the temperature changes observed during the plant experiments and those predicted by the model. Equation (14) shows the problem structure where $R$ is the number of experiments performed, $\Delta \mathbf{T}_{r}^{o b s}$ and $\Delta \mathbf{T}_{r}^{E C}$ are the $N$-dimensional TWT changes observed during the $r^{\text {th }}$ experiment and predicted by the EC-SMR model, respectively, and $\Delta p_{j, r}$ is the valve adjustment for the $j^{\text {th }}$ valve in the $r^{\text {th }}$ experiment. All the $6 M$ decision variables are positive variables.

$$
\begin{aligned}
& \min _{\overline{\boldsymbol{\Delta f}}, \boldsymbol{\beta}, \boldsymbol{\sigma}_{\mathbf{x}}, \boldsymbol{\sigma}_{\mathbf{y}}, \mathbf{k}_{\mathbf{x}}, \mathbf{k}_{\mathbf{y}}} \sum_{r=1}^{R}\left\|\boldsymbol{\Delta} \mathbf{T}_{r}^{o b s}-\boldsymbol{\Delta} \mathbf{T}_{r}^{E C}\right\|^{2} \\
& \text { s.t. } \Delta T_{i, r}^{E C}=\sum_{j=1}^{M} \Delta p_{j, r}\left(\overline{\Delta f}_{j} \beta_{j} \Phi_{i j}+\sum_{k=1, k \neq j}^{M}-\frac{\overline{\Delta f}_{j}}{M-1} \beta_{k} \Phi_{i k}\right) \\
& \forall i \in\{1,2, \ldots, N\}, \forall r \in\{1,2, \ldots, R\} \\
& \overline{\Delta f}_{j}, \beta_{j}, \sigma_{x j}, \sigma_{y j}, k_{x j}, k_{y j}>0 \quad \forall j \in\{1,2, \ldots, M\}
\end{aligned}
$$

In Eq. (14), the parameters $\overline{\Delta f}_{j}$ and $\beta_{k} \forall b_{j}, b_{k}$ are always present in pairs. Since no fuel-specific equality constraint is imposed, such as the total fuel input to the furnace, this leads to non-uniqueness, as per the stated problem formulation, of $\overline{\Delta f}_{j}$ and $\beta_{k}$, i.e., if $\overline{\Delta f}_{j}{ }^{*}$ and $\beta_{k}{ }^{*}$ are the estimated optimal parameters, then for any real number $\alpha>0, \alpha \overline{\Delta f}_{j}{ }^{*}$ and $\beta_{k}{ }^{*} / \alpha$ are also a valid optimal solution to the parameter estimation problem. To avoid this the model is reformulated using the following parametric definitions,

$$
\begin{aligned}
& a_{j}=\overline{\Delta f}_{j} \times \beta_{j} \quad \forall j \in\{1,2, \ldots, M\} \\
& r_{j}=\frac{\beta_{j}}{\beta_{1}}
\end{aligned}
$$

$a_{j}$ acts as the counterpart of $\beta_{j}$ with respect to valve adjustments, and thus allows expressing the model explicitly in terms of valve openings rather than fuel throughput changes. Also $\frac{1}{\sigma_{x}^{2}}$ and $\frac{k_{x}}{\sigma_{x}^{2}}$ are directly treated as decision variables instead of $\sigma_{x}$ and $k_{x}$ 
to avoid unnecessary nonlinearities (similarly for $\sigma_{y}$ and $k_{y}$ ). Let $\frac{1}{\sigma_{x}^{2}}, \frac{k_{x}}{\sigma_{x}^{2}}, \frac{1}{\sigma_{y}^{2}}$ and $\frac{k_{y}}{\sigma_{y}^{2}}$ be represented by $\theta_{x}, \phi_{x}, \theta_{y}$, and $\phi_{y}$, respectively. Then Eq. 14 is reformulated to,

$$
\begin{aligned}
& \min _{\mathbf{a}, \boldsymbol{r}, \boldsymbol{\theta}_{\mathbf{x}}, \boldsymbol{\phi}_{\mathbf{x}}, \boldsymbol{\theta}_{\mathbf{y}}, \boldsymbol{\phi}_{\mathbf{y}}} \sum_{r=1}^{R}\left\|\boldsymbol{\Delta} \mathbf{T}_{r}^{o b s}-\Delta \mathbf{T}_{r}^{E C}\right\|^{2} \\
& \text { s.t. } \\
& \qquad \begin{aligned}
\Delta T_{i, r}^{E C} & =\sum_{j=1}^{M} \Delta p_{j, r}\left(a_{j} \Phi_{i j}+\sum_{k=1, k \neq j}^{M} a_{k j} \Phi_{i k}\right) \\
& =\sum_{j=1}^{M} \Delta p_{j, r} \sum_{k=1}^{M} a_{k j} \Phi_{i k} \\
& \forall i \in\{1,2, \ldots, N\}, \forall r \in\{1,2, \ldots, R\} \\
r_{1} & =1 \\
a_{j}, r_{j}, \theta_{x j}, \phi_{x j}, \theta_{y j}, \phi_{x j}>0 & \forall j \in\{1,2, \ldots, M\}
\end{aligned}
\end{aligned}
$$

In Eq. 16, $a_{k j}=-\frac{\overline{\Delta f}}{M-1} \beta_{k}=-\frac{a_{j}}{M-1} \frac{r_{k}}{r_{j}}$ and $a_{j j}=a_{j}$. The $r_{1}=1$ specification arises out of the parametric definition in Eq. 15. Note that any other, $\beta$ could have been used to define the normalized parameters $r$. The reformulation leads to one less degree of freedom resulting from elimination of inherent redundancy in the original formulation. Also note that Eq. (16) represents the limiting case of EC-SMR model where linear approximations for various interactions in the furnace system are made, and the EC-SMR model reduces to a form similar to Eq. (4), with $\sum_{k=1}^{M} a_{k j} \Phi_{i k}$ being analogous to $g_{i j}$, though with far fewer fitted parameters requiring estimation from plant data (i.e. $6 M-1$, not $N \times M)$.

The reformulated nonlinear optimization problem was solved using CONOPT $^{25}$ in the GAMS $^{26}$ environment. Reasonable lower and upper bounds were imposed on the decision variables, e.g., the upper bound for $\theta_{x j}$ (consequently, the lower bound on $\sigma_{x j}$ ) was set such that the direct effect of burner $b_{j}$ (Figure 6(b) reaches at least the nearest tube row. The solution time was $\sim 4$ hours on a Windows PC with a $2.8 \mathrm{GHz}$, Intel Core i5 processor, and 8 GB RAM. Note that the parameter estimation is not expected to be performed in real-time. The model update/parameter re-estimation may be performed occasionally if the plant undergoes a major change such as blockage 
of a few burners or tubes, that substantially changes the flue-gas flow pattern and consequently, the temperature distribution. However, once the model parameters have been estimated, the model can be used for furnace temperature optimization in real-time because the run time of the furnace optimization program (described in the Section 4.2 is only a few minutes, which is shorter than the time constant of the process itself.

\section{Results and Discussion}

\subsection{Model Predictions vs Plant Observations}

A subset of experiments that were not a part of the initial experimental-design and parameter estimation calculations are used to assess the model accuracy. In addition, the EC-SMR model accuracy is compared against that of the PLS model. Though the number of test cases is limited, some important insights into the performance of these models can still be obtained. The prediction accuracy $(\epsilon)$ for each test-experiment is quantified as follows,

$$
\epsilon=\left[1-\frac{\sum_{i=1}^{N} \frac{\left|\Delta T_{i, o b s}-\Delta T_{i, \text { pred }}\right|}{\left|\Delta T_{i, \text { obs }}\right|} \times\left(\left|\Delta T_{i, \text { obs }}\right|+\left|\Delta T_{i, \text { pred }}\right|\right)}{\sum_{i=1}^{N}\left|\Delta T_{i, \text { obs }}\right|+\left|\Delta T_{i, \text { pred }}\right|}\right] \times 100
$$

Eq. (17) takes a weighted sum of the relative error for each tube, where the weight is proportional to the sum of the magnitude of the observed $\left(\Delta T_{i, o b s}\right)$ and predicted changes $\left(\Delta T_{i, p r e d}\right)$. Thus a lower weight is assigned to the tubes for which the observed and predicted changes are low. This avoids unreasonably large relative error contribution from the tubes for which $\left|\Delta T_{i, o b s}\right|$ and the errors $\left(\left|\Delta T_{i, \text { obs }}-\Delta T_{i, p r e d}\right|\right)$ are small but the relative errors $\left(\frac{\left|\Delta T_{i, o b s}-\Delta T_{i, p r e d}\right|}{\left|\Delta T_{i, o b s}\right|}\right)$ are large. As defined, $\epsilon$ can be less than $-100 \%$.

While Table 1 shows the accuracy of the model, the color-coded plots are again used for improved visualization. Figures 7 and 8 separately compare the model predictions against the observations for test-experiment 1 . 

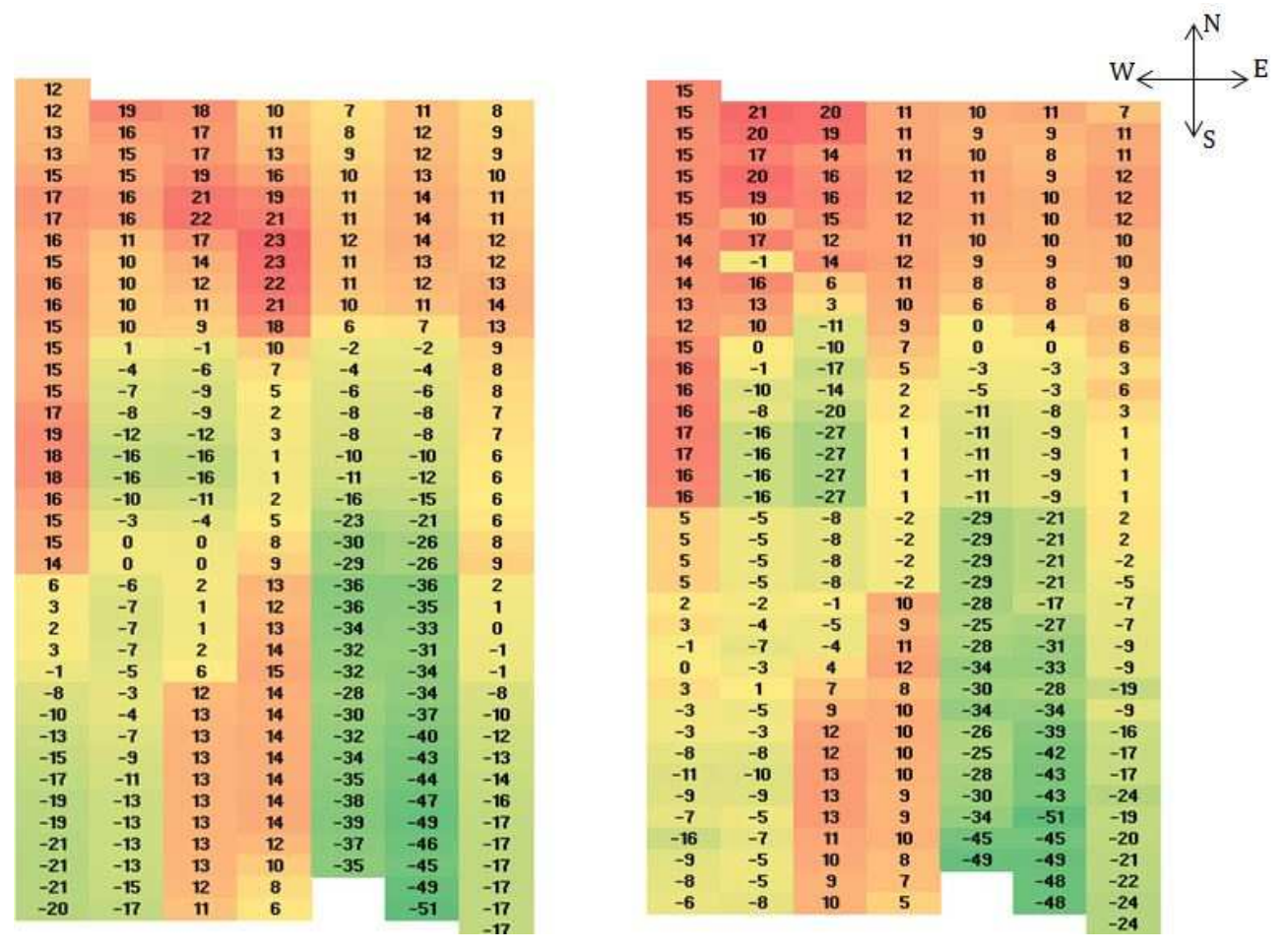

Figure 7: Comparison of tube-wall temperature changes (in $K$ ) observed (right) and those predicted by the EC-SMR model (left) for the test-experiment 1 . The reported values are a uniformly scaled version of actual measurements.

Although the reported values are scaled version of the actual values, they can still be compared as they have been scaled uniformly. Also, $\epsilon$ depends neither upon the scale-factor used to transform the data nor the temperature units used. Figure 7 shows that the EC-SMR model has been able to capture the re-distribution effects, which is crucial for balancing the furnace at the level of valve manipulations, rather than fuelthroughput changes of individual burners. Similar performance is observed for the other test-experiments (see the figures in Appendix B). Comparison of Figures 7 and 8 (along with those in Appendix B clearly shows superior performance of the EC-SMR model. A significant advantage of the EC-SMR model over the PLS model is that it gives more spatially 'smooth' predictions for TWT changes. In Figure 8 a number of regions can be observed where significant differences in the temperature changes 

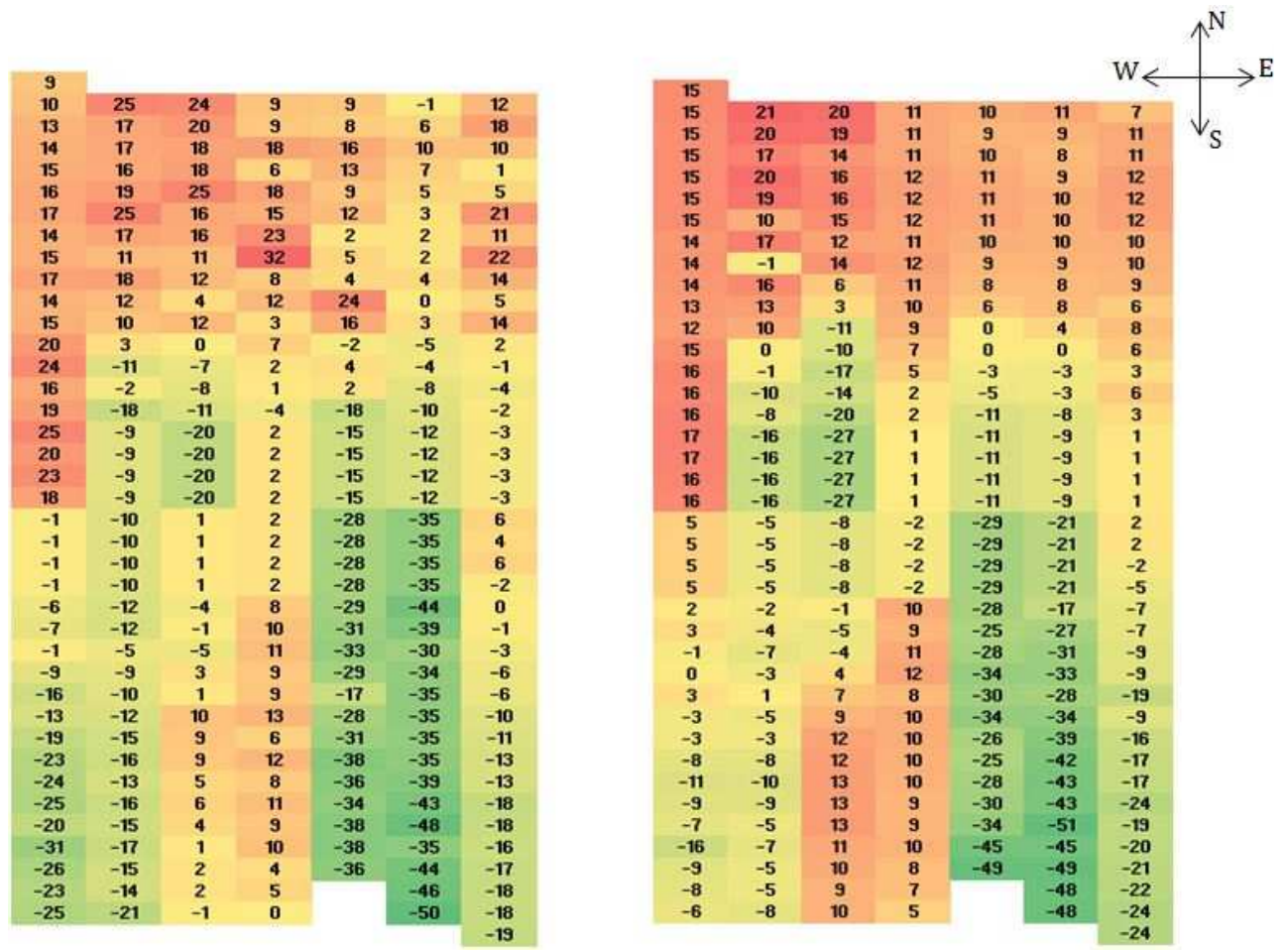

Figure 8: Comparison of tube-wall temperature changes (in $K$ ) observed (right) and those predicted by the PLS model (left) for the test-experiment 1 . The reported values are a uniformly scaled version of actual measurements.

for neighboring (along a tube row) tubes have been predicted, which is not reasonable. Such 'non-smooth' behavior for OLS or PLS model predictions can be attributed to the lack of data or the number of experiments in the training set used to fit the parameters. As was shown in Figure 11(b) in Appendix A, the plant measurements are subject to corruption due to various disturbances, leading to abrupt differences in neighboring TWT changes. Such incorrect behavior is then manifested in the gains, $g_{i j}$, estimated by the PLS or OLS model, which then cause 'non-smooth' predictions. The EC-SMR model, by virtue of its fuel-redistribution-based formulation, is robust to such noise in the data, and thus gives spatially smoother predictions. This noise-filtering property of the EC-SMR model can also be leveraged for those scenarios where the temperature gains are directly measured by manipulating the valves one at a time during data col- 
lection. Although the $\epsilon_{s}$ for the EC-SMR model remain $\sim 50 \%$, it can be implemented

\begin{tabular}{lcc} 
& EC-SMR model & PLS model \\
\hline Test-experiment 1 (see Figures 7 and 8) & $50 \%$ & $37 \%$ \\
Test-experiment 2 (see Figures B.12]and B.13) & $55 \%$ & $59 \%$ \\
Test-experiment 3 (see Figures B.14 and B.15) & $50 \%$ & $47 \%$ \\
\hline
\end{tabular}

Table 1: Accuracy $(\epsilon)$ comparisons between the EC-SMR and PLS model predictions

in a multistage scheme where the EC-SMR model based furnace-balancing program (discussed in the next subsection) is re-run to compute a revised set of valve manipulations once steady-state measurements have been obtained after an initial estimate of optimal valve adjustments. Since the EC-SMR model provides an estimate of the combined effects due to individual burners, unlike a PLS model, employing small manipulations of smaller subset of valves (fine-balancing) can be implemented after an initial 'coarse-balancing'.

\subsection{Optimal Furnace Balancing}

In Section 2 the importance of minimizing the TWT non-uniformity and the maximum TWT was discussed. The non-uniformity can be characterized by the standarddeviation or the range, defined as the difference between the maximum and minimum tube-wall temperature, of the TWT measurements. Ideally, furnace balancing includes some weighted combination of the non-uniformity criteria and the maximum of the TWT distribution as an objective function to be minimized. The precise weights will depend on the economic benefits realized through reduction in these values, and can be estimated through detailed process simulation of the complete hydrogen plant. While it is expected that a reduction in value of one factor will lead to reduction in the value of the other, it is worthwhile to do a comparative analysis through implementation of different furnace-balancing schemes. Three furnace balancing schemes are presented here. In scheme 1 (Eq. (18)) the sample standard-deviation of the TWT distribution is minimized, in scheme 2 (Eq. (19)) the maximum tube-wall temperature is minimized using a min-max formulation, and in scheme 3 (Eq. (20) the range is minimized. Note that the constraints $T_{\nu} \geq T_{i}$ and $T_{\mu} \leq T_{i}$ in Eq. (20) ensure that at the optimal 
point, $T_{\nu}$ and $T_{\mu}$ reflect the maximum and the minimum of all the TWTs, respectively. Similarly in Eq. 19].

Scheme 1:

$$
\begin{array}{ll}
\min _{\Delta \mathbf{p}, y_{1}, y_{2}, \ldots, y_{M}} & \sum_{i=1}^{N}\left(T_{i}-T_{a v g}\right)^{2} \\
\text { s.t. } \quad & T_{i}=T_{i, o}+\sum_{j=1}^{M} \Delta p_{j} \sum_{k=1}^{M} a_{k j} \Phi_{i k} \quad \forall i \in\{1,2, \ldots, N\} \\
& T_{a v g}=\sum_{i=1}^{N} T_{i} / N \\
& \sum_{j=1}^{M} y_{j} \leq n \\
& \Delta p_{j} \leq U y_{j} \quad \forall j \in\{1,2, \ldots, M\} \\
\Delta p_{j} \geq L y_{j} \quad \forall j \in\{1,2, \ldots, M\} \\
y_{j} \in\{0,1\} \quad \forall j \in\{1,2, \ldots, M\}
\end{array}
$$

Scheme 2:

$$
\begin{array}{cl}
\min _{\Delta \mathbf{p}, y_{1}, y_{2}, \ldots, y_{M}} & T_{\nu} \\
\text { s.t. } & T_{\nu} \geq T_{i} \quad \forall i \in\{1,2, \ldots, N\} \\
& T_{i}=T_{i, o}+\sum_{j=1}^{M} \Delta p_{j} \sum_{k=1}^{M} a_{k j} \Phi_{i k} \quad \forall i \in\{1,2, \ldots, N\} \\
& \sum_{j=1}^{M} y_{j} \leq n \\
& \Delta p_{j} \leq U y_{j} \quad \forall j \in\{1,2, \ldots, M\} \\
& \Delta p_{j} \geq L y_{j} \quad \forall j \in\{1,2, \ldots, M\} \\
& y_{j} \in\{0,1\} \quad \forall j \in\{1,2, \ldots, M\}
\end{array}
$$


Scheme 3:

$$
\begin{array}{cl}
\min _{\Delta \mathbf{p}, y_{1}, y_{2}, \ldots, y_{M}} & T_{\nu}-T_{\mu} \\
\text { s.t. } \quad & T_{\nu} \geq T_{i} \quad \forall i \in\{1,2, \ldots, N\} \\
& T_{\mu} \leq T_{i} \quad \forall i \in\{1,2, \ldots, N\} \\
& T_{i}=T_{i, o}+\sum_{j=1}^{M} \Delta p_{j} \sum_{k=1}^{M} a_{k j} \Phi_{i k} \quad \forall i \in\{1,2, \ldots, N\} \\
& \sum_{j=1}^{M} y_{j} \leq n \\
& \Delta p_{j} \leq U y_{j} \quad \forall j \in\{1,2, \ldots, M\} \\
& \Delta p_{j} \geq L y_{j} \quad \forall j \in\{1,2, \ldots, M\} \\
& y_{j} \in\{0,1\} \quad \forall j \in\{1,2, \ldots, M\}
\end{array}
$$

In Eqs. [18-20), $T_{i, o}$ denotes the initial temperature of the tube $\tau_{i}$. The upper bounds $(U)$ on $\Delta p_{j}$ are precautionary measures to prevent flame de-stabilization. In practice, it is expected that there would be some upper bound $(n)$, based on plant-operator preferences, on the number of valves that are allowed to undergo manipulations simultaneously. Moreover, restrictions can be imposed on the minimum valve-position change $(L)$ for valves that undergo manipulations. $y_{j}$ is a binary variable denoting whether burner $b_{j}$ is manipulated $\left(y_{j}=1\right)$ or not $\left(y_{j}=0\right)$. These mixed-integer optimal-programs were solved (with $L, U$, and $n$ as 5,25 , and 32 , respectively) using IBM ILOG CPLEX 12.6 in GAMS. Figure 9 shows the resulting predicted TWT distribution for relatively balanced furnaces. As expected, all the three schemes lead to reduction in the standard-deviation, range, and the maximum temperature, with scheme 1 producing the smallest standard-deviation while scheme 2 leads to the lowest maximum temperature. While scheme 1 manages to bring both the maximum and minimum temperatures closer to the average temperature, scheme 2 trades off on the lower end of the distribution to achieve the lowest maximum TWT.

Scheme 1 was tested on the test-bed industrial furnace with a slight adaptation that the valves were manipulated in groups of four. The calculation run time was $\sim 1$ minute. Figure 10 shows the TWT distribution histogram for the furnace before and 


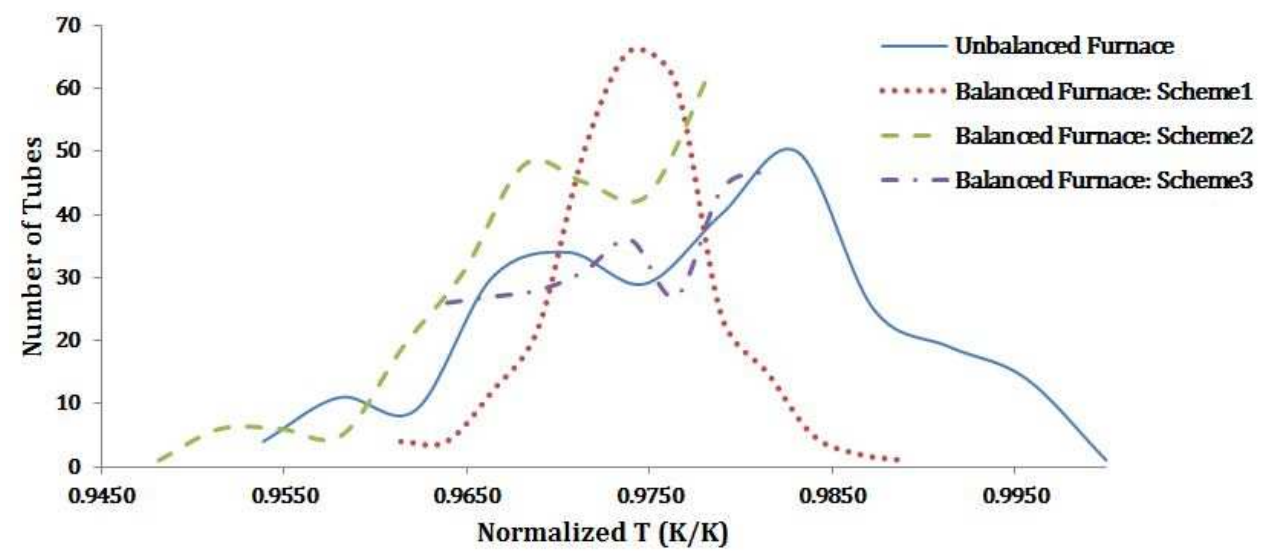

Figure 9: Comparison of predicted TWT distribution after application of two different furnace balancing schemes using the EC-SMR model. The reported temperatures have been normalized using the measured maximum TWT for the unbalanced furnace. Note that the shown temperature distributions for the balanced furnaces in this figure refer to those obtained from the model.

after balancing. A $44 \%$ reduction in the standard deviation was obtained. The TWT distribution shown in Figure 10 is different from that shown in the Figure 4 which emphasizes the non-static nature of the temperature distribution. Note that at the initiation of the furnace balancing program implementation, a few tubes showed unreasonably high temperatures and thus were removed from the balancing scheme. Understandably, this is one of the limitations of data-driven SMR models which does not take into account localized effects, at the level of an individual tube, such as abnormally reduced catalyst activity within a reformer tube if these effects are not included in the training data used for building the model. Manual intervention is necessary when such effects occur.

While the EC-SMR model based furnace balancing facilitates the reduction of temperature non-uniformity in the furnace, substantial benefits can be expected upon increasing the furnace temperature as noted previously. Future publications will cover the details on the potential benefits and the deployment of the furnace optimization solution through the Smart Manufacturing platform which is currently under development 27 . 


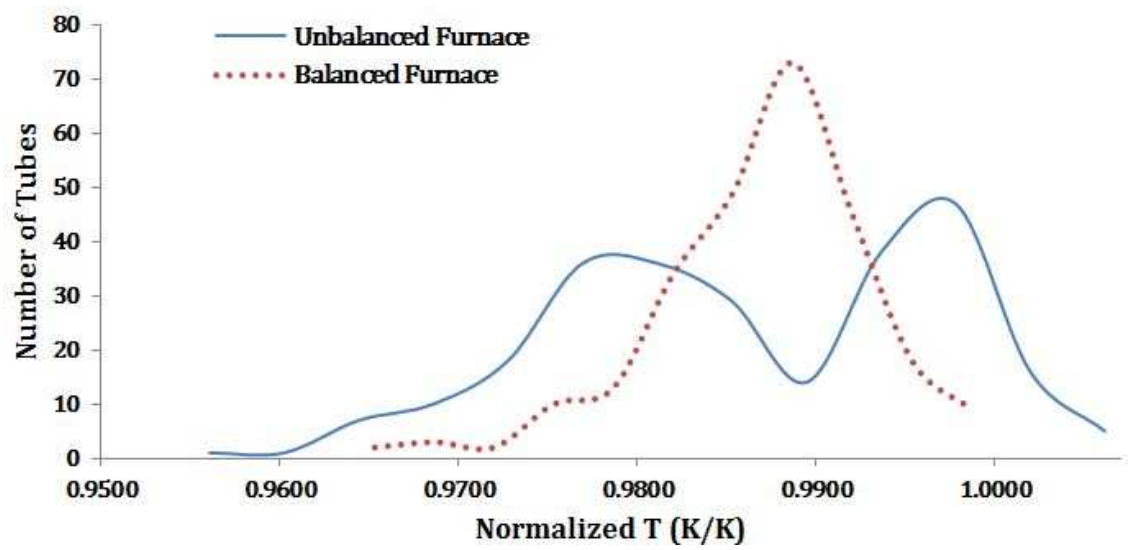

Figure 10: Comparison of TWT distribution after application EC-SMR model based furnace balancing scheme. The normalization factor used in Figure 4 has been used here. Note that the shown temperature distributions in this figure reflect data from an actual operating furnace. 


\section{Conclusions}

In this work, a novel data-driven reduced-order model, termed as egg-crate SMR (EC-SMR) model, is used to describe the operation of an industrial-scale SMR furnace. The proposed model name, Egg-Crate SMR, is inspired by the methodology adopted in this work, where the local and long-range effects of burner fuel-stream valve manipulations are accounted for separately. The fuel-redistribution based formulation renders the EC-SMR model robust to data corruption and allows for easy incorporation of nonlinear interactions, if desired. Being computationally efficient, it is suitable for real-time furnace temperature distribution optimization. Owing to the superior performance by the EC-SMR model compared to the PLS model, it was employed in a few different schemes for furnace-balancing. These schemes were used to understand the trade-offs between minimization of standard-deviation and the maximum value of the TWT distribution. A $44 \%$ reduction in temperature nonuniformity was obtained upon testing with the standard-deviation based optimization scheme on a commercial testbed furnace. In the future, a hybrid combination of the EC-SMR and the CFD-based SMR model, obtained by imposing several patterns inferred from the CFD model on the EC-SMR model, and the benefits realized upon the furnace temperature adjustment through the Smart Manufacturing platform will be reported.

\section{Acknowledgment}

The authors gratefully acknowledge the financial support from the U.S. Department of Energy through grant DE-EE0005763/00011 "Industrial Scale Demonstration of Smart Manufacturing Achieving Transformational Energy Productivity Gains”.

\section{Disclaimer}

This report was prepared as an account of work sponsored by an agency of the United States Government. Neither the United States Government nor any agency thereof, nor any of their employees, makes any warranty, expressed or implied, or assumes any legal liability or responsibility for the accuracy, completeness, or usefulness 
of any information, apparatus, product, or process disclosed, or represents that its use would not infringe privately owned rights. Reference herein to any specific commercial product, process, or service by trade name, trademark, manufacturer, or otherwise does not necessarily imply its endorsement, recommendation or favoring by the United States Government or any agency thereof. The views and opinions of authors expressed herein do not necessarily state or reflect those of the United States Government or any agency thereof. 


\section{Appendix A. TWT temperature changes}

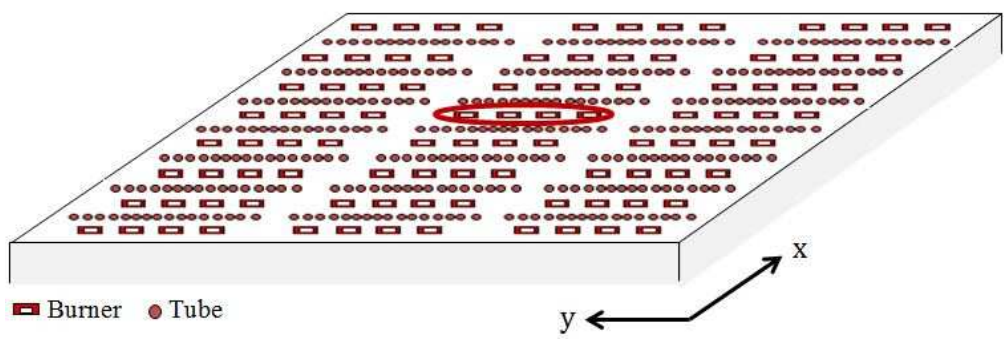

(a)

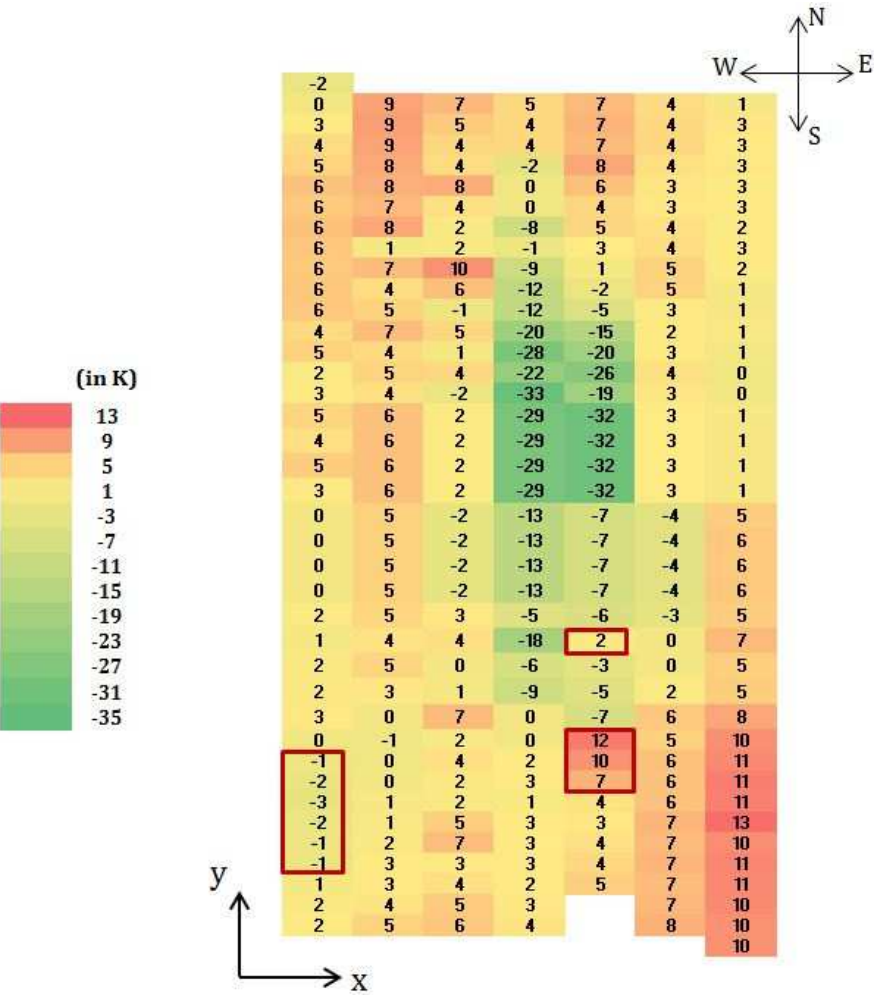

(b)

Figure A.11: (a) Encircled burners are closed partially inducing the temperature changes as shown in (b) The reported temperatures are a uniformly scaled version of actual values. The boxed readings in (b) are 'suspicious' values, probably resulting from process fluctuations and camera movements. 
Appendix B. Comparison of model prediction against plant observations

\begin{tabular}{|c|c|c|c|c|c|c|}
\hline 16 & & & & & & \\
\hline 16 & -3 & -24 & -19 & -31 & -9 & 9 \\
\hline 16 & -4 & -25 & -20 & -36 & -10 & 10 \\
\hline 16 & -5 & -24 & -21 & -40 & -10 & 10 \\
\hline 16 & -5 & -21 & -19 & -43 & -9 & 11 \\
\hline 16 & -4 & -19 & -15 & -46 & -7 & 13 \\
\hline 15 & -3 & -16 & -13 & -47 & -6 & 13 \\
\hline 15 & -3 & -16 & -8 & -45 & -4 & 15 \\
\hline 14 & -3 & -18 & -8 & -43 & -4 & 15 \\
\hline 16 & -3 & -20 & -8 & -41 & -4 & 16 \\
\hline 16 & -3 & -21 & -8 & -39 & -4 & 17 \\
\hline 16 & -2 & -21 & -9 & -40 & -7 & 16 \\
\hline 17 & -4 & -16 & -3 & -26 & -8 & 12 \\
\hline 18 & -6 & -16 & -2 & -22 & -8 & 11 \\
\hline 19 & -7 & -15 & -1 & -18 & -8 & 10 \\
\hline 22 & -7 & -13 & -1 & -15 & -8 & 10 \\
\hline 23 & -10 & -13 & 2 & -12 & -8 & 9 \\
\hline 24 & -11 & -12 & 4 & -7 & -8 & 8 \\
\hline 24 & -11 & -12 & 4 & -9 & -10 & 8 \\
\hline 21 & -5 & -7 & 5 & -13 & -13 & 8 \\
\hline 19 & 2 & -1 & 8 & -21 & -19 & 8 \\
\hline 19 & 6 & 4 & 11 & -28 & -25 & 11 \\
\hline 18 & 6 & 4 & 12 & -27 & -24 & 12 \\
\hline 20 & 8 & 7 & 17 & -33 & -30 & 15 \\
\hline 19 & 7 & 6 & 16 & -32 & -28 & 15 \\
\hline 20 & 8 & 6 & 17 & -31 & -26 & 16 \\
\hline 23 & 10 & 7 & 18 & -29 & -23 & 16 \\
\hline 20 & 14 & 11 & 19 & -25 & -19 & 17 \\
\hline 18 & 19 & 17 & 18 & -18 & -14 & 18 \\
\hline 18 & 20 & 18 & 18 & -19 & -14 & 19 \\
\hline 19 & 21 & 19 & 18 & -21 & -16 & 20 \\
\hline 19 & 22 & 19 & 18 & -22 & -18 & 21 \\
\hline 18 & 22 & 19 & 18 & -22 & -19 & 22 \\
\hline 19 & 23 & 20 & 18 & -25 & -22 & 22 \\
\hline 20 & 23 & 19 & 18 & -26 & -25 & 22 \\
\hline 19 & 23 & 19 & 16 & -24 & -23 & 21 \\
\hline 19 & 22 & 19 & 14 & -23 & -23 & 21 \\
\hline 20 & 22 & 18 & 11 & & -28 & 21 \\
\hline 21 & 23 & 17 & 10 & & -27 & 20 \\
\hline
\end{tabular}

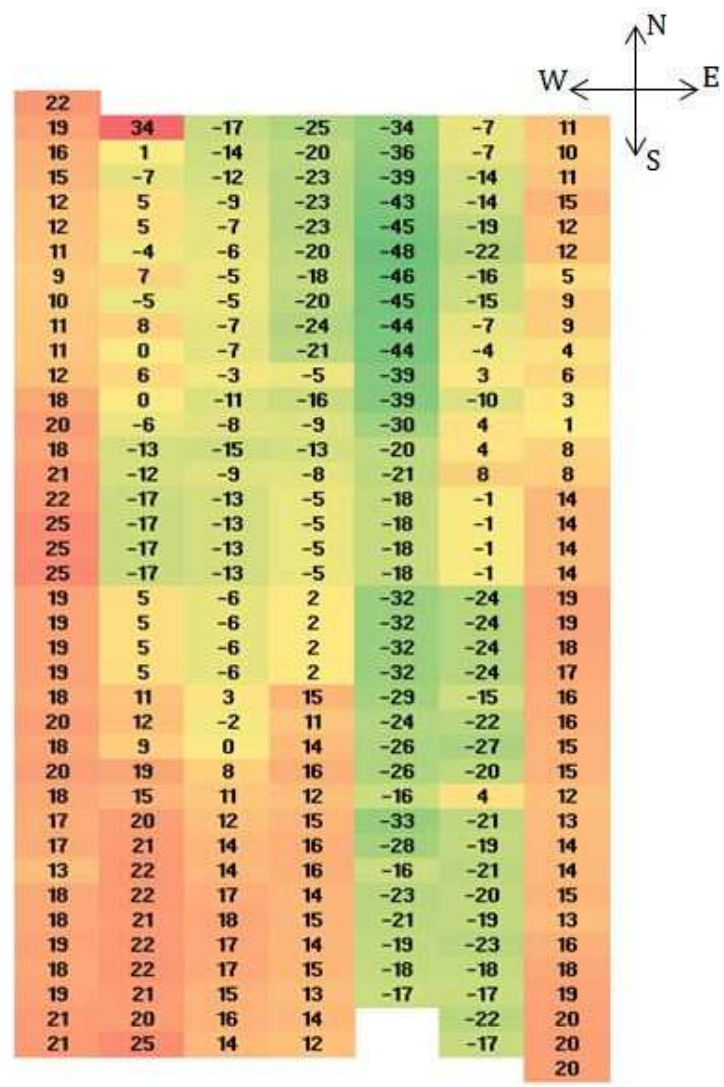

Figure B.12: Comparison of tube-wall temperature changes (in $K$ ) observed (right) and those predicted by the EC-SMR model (left) for the test-experiment 2. The reported values are a uniformly scaled version of actual measurements. 


\begin{tabular}{|c|c|c|c|c|c|c|}
\hline 24 & & & & & & \\
\hline 21 & -3 & -23 & -41 & -37 & -16 & -6 \\
\hline 15 & -5 & -21 & -39 & -40 & -14 & -2 \\
\hline 14 & -6 & -19 & -28 & -41 & -19 & -4 \\
\hline 9 & -6 & -22 & -30 & -49 & -16 & -3 \\
\hline 7 & -9 & -16 & -24 & -50 & -19 & -5 \\
\hline 7 & -1 & -16 & -20 & -52 & -16 & 1 \\
\hline 3 & -5 & -16 & -17 & -47 & -14 & -3 \\
\hline 5 & -4 & -17 & -12 & -50 & -18 & 0 \\
\hline 7 & -1 & -13 & -12 & -46 & -11 & -1 \\
\hline 5 & -4 & -16 & -16 & -41 & -13 & -7 \\
\hline 8 & -4 & -14 & -11 & -36 & -11 & 4 \\
\hline 15 & -4 & -18 & -14 & -43 & -18 & 0 \\
\hline 20 & -11 & -18 & -15 & -29 & -6 & -1 \\
\hline 14 & -11 & -16 & -10 & -26 & -9 & -2 \\
\hline 17 & -16 & -18 & -14 & -29 & 0 & 1 \\
\hline 22 & -13 & -17 & -7 & -23 & -8 & 3 \\
\hline 20 & -13 & -17 & -7 & -23 & -8 & 3 \\
\hline 24 & -13 & -17 & -7 & -23 & -8 & 3 \\
\hline 21 & -13 & -17 & -7 & -23 & -8 & 3 \\
\hline 10 & 3 & 0 & 5 & -33 & -36 & 14 \\
\hline 10 & 3 & 0 & 5 & -33 & -36 & 13 \\
\hline 10 & 3 & 0 & 5 & -33 & -36 & 15 \\
\hline 10 & 3 & 0 & 5 & -33 & -36 & 11 \\
\hline 10 & 4 & 1 & 7 & -30 & -33 & 12 \\
\hline 11 & 4 & 3 & 14 & -29 & -31 & 11 \\
\hline 14 & 8 & 5 & 14 & -29 & -30 & 10 \\
\hline 14 & 10 & 13 & 15 & -26 & -24 & 10 \\
\hline 12 & 10 & 9 & 14 & -20 & -21 & 10 \\
\hline 14 & 16 & 17 & 15 & -22 & -22 & 12 \\
\hline 12 & 16 & 17 & 15 & -27 & -19 & 12 \\
\hline 17 & 16 & 16 & 17 & -26 & -21 & 14 \\
\hline 14 & 22 & 20 & 12 & -27 & -22 & 15 \\
\hline 15 & 19 & 21 & 16 & -25 & -25 & 15 \\
\hline 15 & 22 & 16 & 17 & -28 & -31 & 14 \\
\hline 14 & 24 & 21 & 12 & -26 & -23 & 16 \\
\hline 16 & 24 & 17 & 10 & -26 & -28 & 15 \\
\hline 17 & 24 & 17 & 9 & & -33 & 14 \\
\hline 18 & 24 & 15 & 9 & & -30 & 13 \\
\hline
\end{tabular}

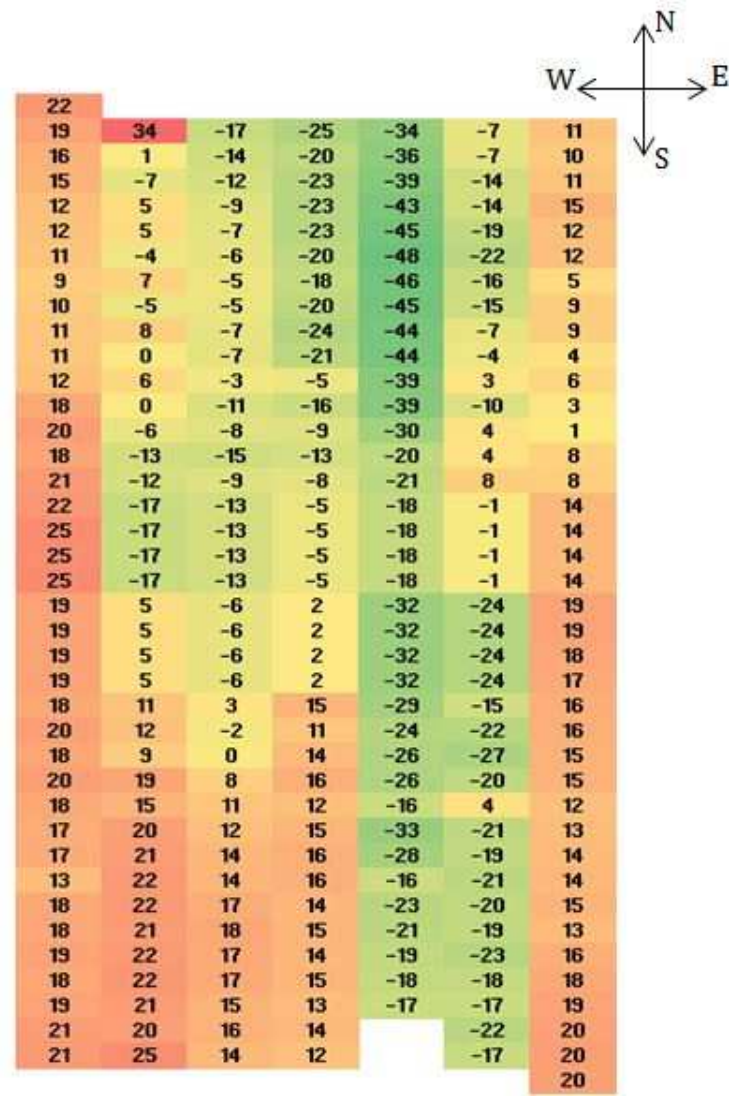

Figure B.13: Comparison of tube-wall temperature changes (in $K$ ) observed (right) and those predicted by the PLS model (left) for the test-experiment 2. The reported values are a uniformly scaled version of actual measurements. 

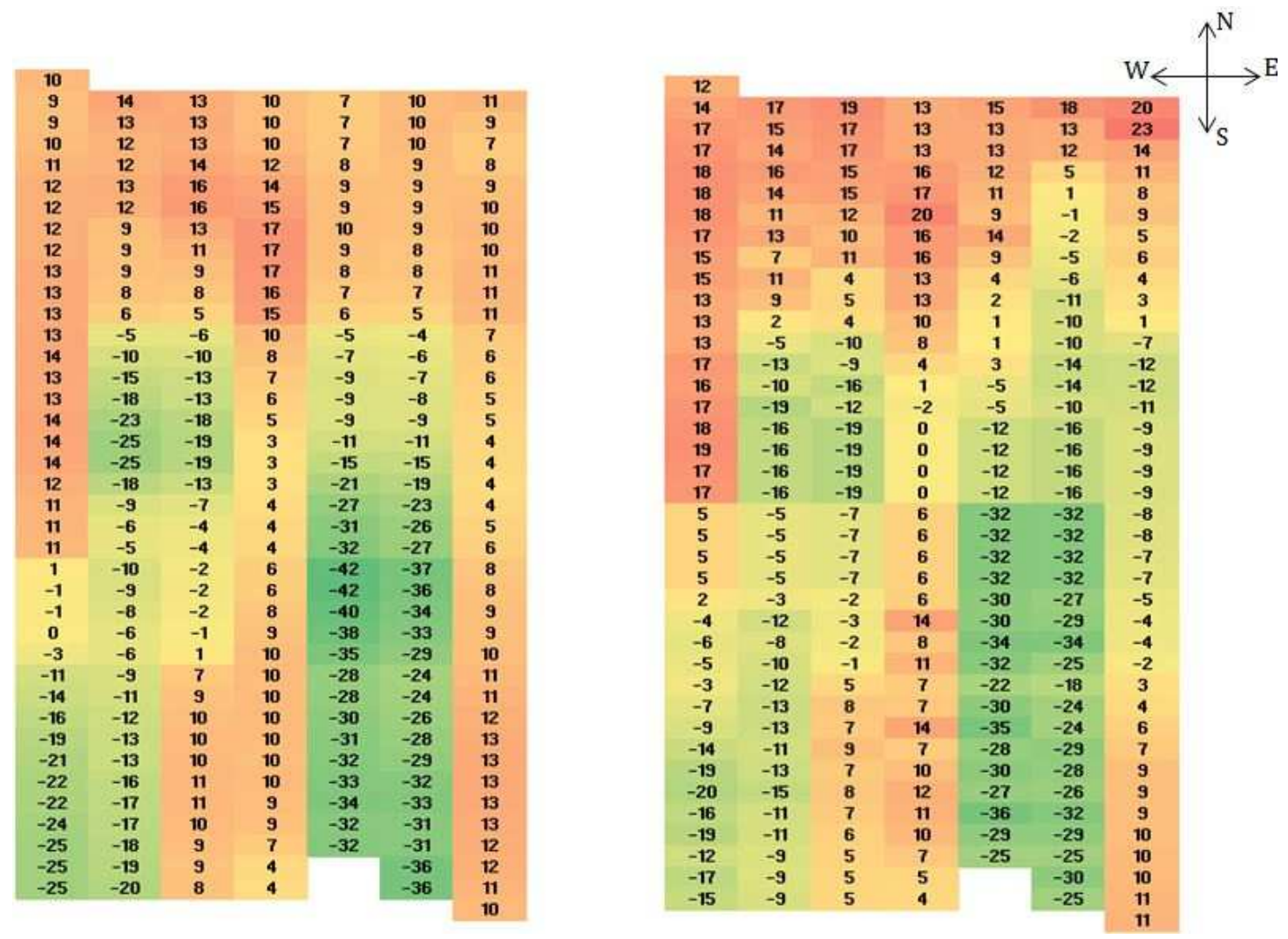

Figure B.14: Comparison of tube-wall temperature changes (in $K$ ) observed (right) and those predicted by the EC-SMR model (left) for the test-experiment 3. The reported values are a uniformly scaled version of actual measurements. 

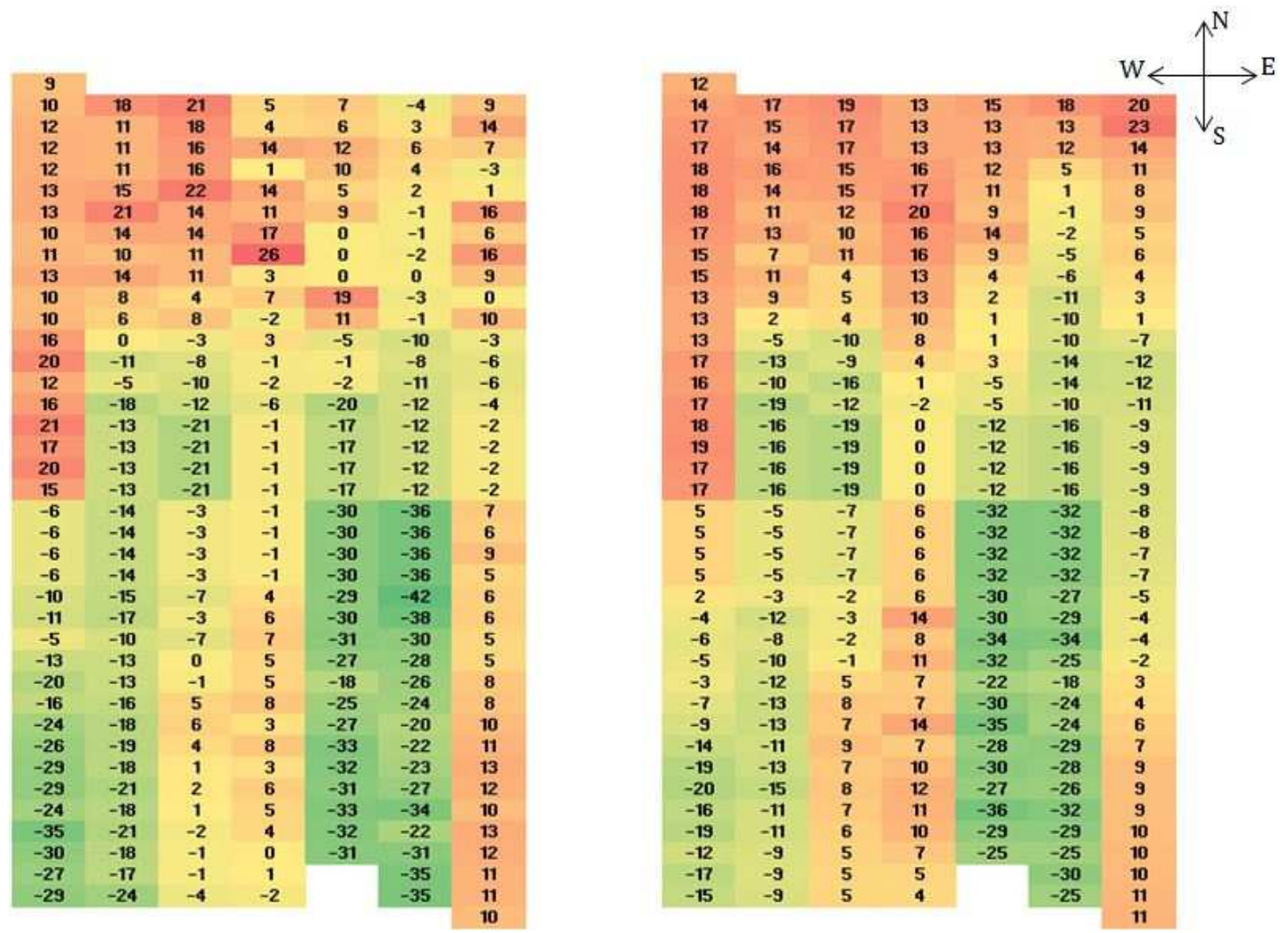

Figure B.15: Comparison of tube-wall temperature changes (in $K$ ) observed (right) and those predicted by the PLS model (left) for the test-experiment 3. The reported values are a uniformly scaled version of actual measurements. 


\section{References}

1. Olivieri, A., Vegliò, F.. Process simulation of natural gas steam reforming: Fuel distribution optimisation in the furnace. Fuel Process Technol 2008;89(6):622632. doi:10.1016/j.fuproc.2007.12.001

2. Esposito, W., Dadebo, S.. Production control utilizing real time optimization. 2011. US Patent 7,881,825.

3. Peng, X.. Analysis of the thermal efficiency limit of the steam methane reforming process. Ind Eng Chem Res 2012;51(50):16385-16392. doi: $10.1021 /$ ie3002843.

4. Murthy, C.V.S., Murthy, M.V.. Modeling and Simulation of a Top-Fired Reformer. Ind Eng Chem Res 1988;27:1832-1840. doi:10.1021/ie00082a016

5. Van Gerven, T., Stankiewicz, A.. Structure, energy, synergy, time-the fundamentals of process intensification. Ind Eng Chem Res 2009;48(5):2465-2474. doi:10.1021/ie801501y.

6. Slavejkov, A.G., Li, X., Joshi, M.L., Waibel, R.T., Bussman, W.. LSV Burner Technology for Improved Efficiency, Reliability and Low-NOx: A Case Study. In: Proceedings of the 51st Annual Safety in Ammonia Plants and Related Facilities Symposium; vol. 53. Vancouver, Canada: AIChE; 2006:.

7. Latham, D.A., McAuley, K.B., Peppley, B.A., Raybold, T.M.. Mathematical modeling of an industrial steam-methane reformer for on-line deployment. Fuel Process Technol 2011;92(8):1574-1586. doi $10.1016 / j$.fuproc.2011.04.001.

8. McGreavy, C., Newmann, M.W.. Development of a mathematical model of a steam methane reformer. In: Proceedings of the Conference on the Industrial Applications of Dynamic Modelling; vol. 57. Durham: Institution of Electrical Engineers: London; 1969:. 
9. Singh, C.P.P., Saraf, D.N.. Simulation of Side Fired SteamHydrocarbon Reformers. Ind Eng Chem Process Des Dev 1979;18(1):1-7. doi:10.1021/i260069a001

10. Zamaniyan, A., Ebrahimi, H., Mohammadzadeh, J.S.S.. A unified model for top fired methane steam reformers using three-dimensional zonal analysis. Chem Eng Process 2008;47(5):946-956. doi:10.1016/j.cep.2007.03.005

11. Dunn, A.J., Yustos, J., Mujtaba, I.M.. Modelling and Simulation of a TopFired Primary Steam Reformer using gPROMS. Dev Chem Eng Miner Process 2008;10(1-2):77-87. doi:10.1002/apj.5500100107.

12. Heynderickx, G.J., Oprins, A.J.M., Marin, G.B., Dick, E.. Threedimensional flow patterns in cracking furnaces with long-flame burners. AIChE J 2001;47(2):388-400. doi:10.1002/aic.690470215.

13. Farnell, R., Cotton, W.. Flue-gas flow patterns in top-fired steam reforming furnaces. Ammonia Plant Safety and Related Facilities 2000;40:144-158.

14. Oprins, A.J.M., Heynderickx, G.J., Marin, G.B.. Three-Dimensional Asymmetric Flow and Temperature Fields in Cracking Furnaces . Ind Eng Chem Res 2001;40(23):5087-5094. doi:10.1021/ie0010114.

15. Zheng, D., Wu, B., Fleitz, J., Trajkovski, R., Zhou, C.Q.. CFD Simulation of a Hydrogen Reformer Furnace. In: Proceedings of the 14th International Heat Transfer Conference; vol. 3. Washington, DC: ASME. ISBN 978-0-7918-4938-5; 2010:233-244. doi:10.1115/IHTC14-23121.

16. Blevins, T.L., Nixon, M.. Control loop foundation: batch and continuous processes. ISA; 2011. ISBN 978-1-936007-54-7.

17. Kumar, A., Baldea, M., Edgar, T.F., Ezekoye, O.A.. Smart manufacturing approach for efficient operation of industrial steam-methane reformers. Ind Eng Chem Res 2015;54(16):4360-4370. doi:10.1021/ie504087z 
18. Esmaili, A., Licht, W., Li, X., Smith, O.. Method of operating a furnace. 2012. US Patent 8,219,247.

19. Wold, S., Sjöström, M., Eriksson, L.. PLS-regression: a basic tool of chemometrics. Chemometrics and Intelligent Laboratory Systems 2001;58(2):109-130. doi:10.1016/S0169-7439(01)00155-1.

20. Davis, J., Edgar, T., Porter, J., Bernaden, J., Sarli, M.. Smart manufacturing, manufacturing intelligence and demand-dynamic performance. Comput Chem Eng 2012;47:145-156. doi:10.1016/j.compchemeng.2012 .06.037.

21. Venkatasubramanian, V.. Drowning in data: Informatics and modeling challenges in a data-rich networked world. AIChE journal 2009;55(1):2-8. doi:10.1002/aic.11756.

22. Qin, S.J.. Process data analytics in the era of big data. AIChE Journal 2014;60(9):3092-3100. doi:10.1002/aic.14523.

23. Edgar, T.F., Davis, J.F.. Smart process manufacturing-a vision of the future. In: Design for Energy and the Environment: Proceedings of the Seventh International Conference on the Foundations of Computer Aided Process Design. Boca Raton, FL: CRC Press; 2009:149-165.

24. Box, G.E., Hunter, W.G., Hunter, J.S., et al. Statistics for experimenters. 2nd ed. ed.; New Jersey: John Wiley \& Sons; 1978. ISBN 0-471-71813-0.

25. Drud, A.. CONOPT: A GRG code for large sparse dynamic nonlinear optimization problems. Mathematical Programming 1985;31(2):153-191. doi:10.1007/BF02591747.

26. Rosenthal, R.E.. GAMS - A User's Guide. 2014. Washington. DC: GAMS Development Corporation.

27. Korambath, P., Wang, J., Kumar, A., Hochstein, L., Schott, B., Graybill, R., Baldea, M., Davis, J.. Deploying Kepler Workflows as Services on 
a Cloud Infrastructure for Smart Manufacturing. Procedia Computer Science

2014;29:2254-2259. doi $10.1016 /$ j.procs.2014 .05.210 